\title{
ER stress regulates myeloid-derived suppressor cell fate through TRAIL-R-mediated apoptosis
}

\author{
Thomas Condamine, ${ }^{1}$ Vinit Kumar, ${ }^{1}$ Indu R. Ramachandran, ${ }^{1}$ Je-In Youn, ${ }^{1}$ Esteban Celis, ${ }^{2}$ \\ Niklas Finnberg, ${ }^{3}$ Wafik S. El-Deiry, ${ }^{3}$ Rafael Winograd, ${ }^{4}$ Robert $\mathrm{H}$. Vonderheide, ${ }^{4}$ \\ Nickolas R. English, ${ }^{5}$ Stella C. Knight, ${ }^{5}$ Hideo Yagita, ${ }^{6}$ Judith C. McCaffrey, ${ }^{2}$ Scott Antonia, ${ }^{2}$ \\ Neil Hockstein,7 Robert Witt, ${ }^{7}$ Gregory Masters, ${ }^{7}$ Thomas Bauer,7 and Dmitry I. Gabrilovich ${ }^{1}$

\begin{abstract}
${ }^{1}$ The Wistar Institute, Philadelphia, Pennsylvania, USA. ${ }^{2} \mathrm{H}$. Lee Moffitt Cancer Center and Research Institute, Tampa, Florida, USA. ${ }^{3}$ Penn State Hershey Cancer Institute, Hershey, Pennsylvania, USA. ${ }^{4}$ Abramson Family Cancer Research Institute,

Perelman School of Medicine, University of Pennsylvania, Philadelphia, Pennsylvania, USA. ${ }^{5}$ Antigen Presentation Research Group Imperial College London, London, United Kingdom. ${ }^{6}$ Department of Immunology, Juntendo University School of Medicine, Tokyo, Japan. ${ }^{7}$ Helen F. Graham Cancer Center and Research Institute, Christiana Care Health System, Newark, Delaware, USA.
\end{abstract}

\begin{abstract}
Myeloid-derived suppressor cells (MDSCs) dampen the immune response thorough inhibition of T cell activation and proliferation and often are expanded in pathological conditions. Here, we studied the fate of MDSCs in cancer. Unexpectedly, MDSCs had lower viability and a shorter half-life in tumor-bearing mice compared with neutrophils and monocytes. The reduction of MDSC viability was due to increased apoptosis, which was mediated by increased expression of TNF-related apoptosis-induced ligand receptors (TRAIL-Rs) in these cells. Targeting TRAIL-Rs in naive mice did not affect myeloid cell populations, but it dramatically reduced the presence of MDSCs and improved immune responses in tumor-bearing mice. Treatment of myeloid cells with proinflammatory cytokines did not affect TRAIL-R expression; however, induction of ER stress in myeloid cells recapitulated changes in TRAIL-R expression observed in tumor-bearing hosts. The ER stress response was detected in MDSCs isolated from cancer patients and tumor-bearing mice, but not in control neutrophils or monocytes, and blockade of ER stress abrogated tumor-associated changes in TRAIL-Rs. Together, these data indicate that MDSC pathophysiology is linked to ER stress, which shortens the lifespan of these cells in the periphery and promotes expansion in BM. Furthermore, TRAIL-Rs can be considered as potential targets for selectively inhibiting MDSCs.
\end{abstract}

\section{Introduction}

Myeloid-derived suppressor cells (MDSCs) are widely considered as an important factor regulating immune responses to different pathologic conditions. Accumulation of these cells is a common occurrence in cancer and many other pathologic conditions (1). MDSCs constitute a heterogeneous group of cells consisting primarily of immature myeloid cells with morphological and phenotypic characteristics similar to those of monocytes and polymorphonuclear neutrophils (PMNs) (referred to herein as M-MDSCs and PMN-MDSCs, respectively) (1-3). MDSCs have a distinct gene expression profile and a number of biochemical and functional differences from normal monocytes and PMNs $(4,5)$. Expansion of MDSCs in cancer is controlled by several growth factors and cytokines, with GM-CSF being the most prominent $(6,7)$. However, the fate of MDSCs in tumor-bearing (TB) hosts remains poorly understood. The fact that MDSCs accumulate in large numbers could suggest that these cells have mechanisms protecting them from apoptosis. Indeed, studies have demonstrated several mechanisms that could promote MDSC survival. These mechanisms include TNFR2 signaling, which supports MDSC survival through upregulation of cellular FLICE-inhibitory protein (c-FLIP) and inhibition of caspase- 8 activity (8), signaling mediated via IL- $4 \alpha$ receptor (9), and decreased cell surface expression of FAS receptor, leading to

Conflict of interest: The authors have declared that no conflict of interest exists. Citation for this article: J Clin Invest. 2014;124(6):2626-2639. doi:10.1172/JCI74056. diminished expression of IRF8 and BAX as well as increased levels of BCL-XL (10). MDSCs induced in highly inflammatory settings had increased resistance to FAS-mediated apoptosis (11). On the other hand, Sinha et al. demonstrated the possibility of CTLs killing MDSCs via FAS-FASL-mediated apoptosis (12). Nonetheless, unbiased analysis of the fate of MDSCs in cancer has been lacking. The initial goal of this study was to investigate the kinetics of MDSC homeostasis in different organs in vivo. To our surprise, our data revealed that MDSCs had much shorter lifespan than their counterpart PMNs and monocytes in tumor-free mice. Further investigation demonstrated that this effect was mediated by changes in the expression of TNF-related apoptosis-induced ligand receptors (TRAIL-Rs) caused by ER stress response in these cells.

\section{Results}

MDSC survival in TB mice. To monitor MDSC homeostasis, we administered BrdU to EL4 TB mice for 8 days in drinking water (pulse phase), followed by its withdrawal for 4 days (chase phase). PMNs and PMN-MDSCs (in naive and TB mice, respectively) were defined as CD11 b $\mathrm{b}^{+} 6 \mathrm{G}^{+} \mathrm{Ly} 6 \mathrm{C}^{\mathrm{lo}}$, and monocytes and M-MDSCs as CD11b+Ly6G-Ly6Chi (Supplemental Figure 1A; supplemental material available online with this article; doi:10.1172/ JCI74056DS1). TB mice had a dramatic increase of MDSCs in spleens and peripheral blood (PB), where PMN-MDSCs represented more than $90 \%$ of all MDSCs $(2,13)$. M-MDSCs incorporated $\mathrm{BrdU}$ significantly faster than monocytes (Figure $1 \mathrm{~A})$. PMN- 
A

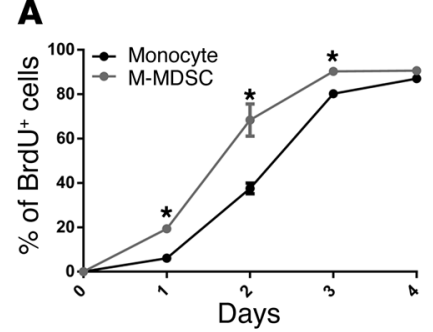

B

C

Blood

Spleen
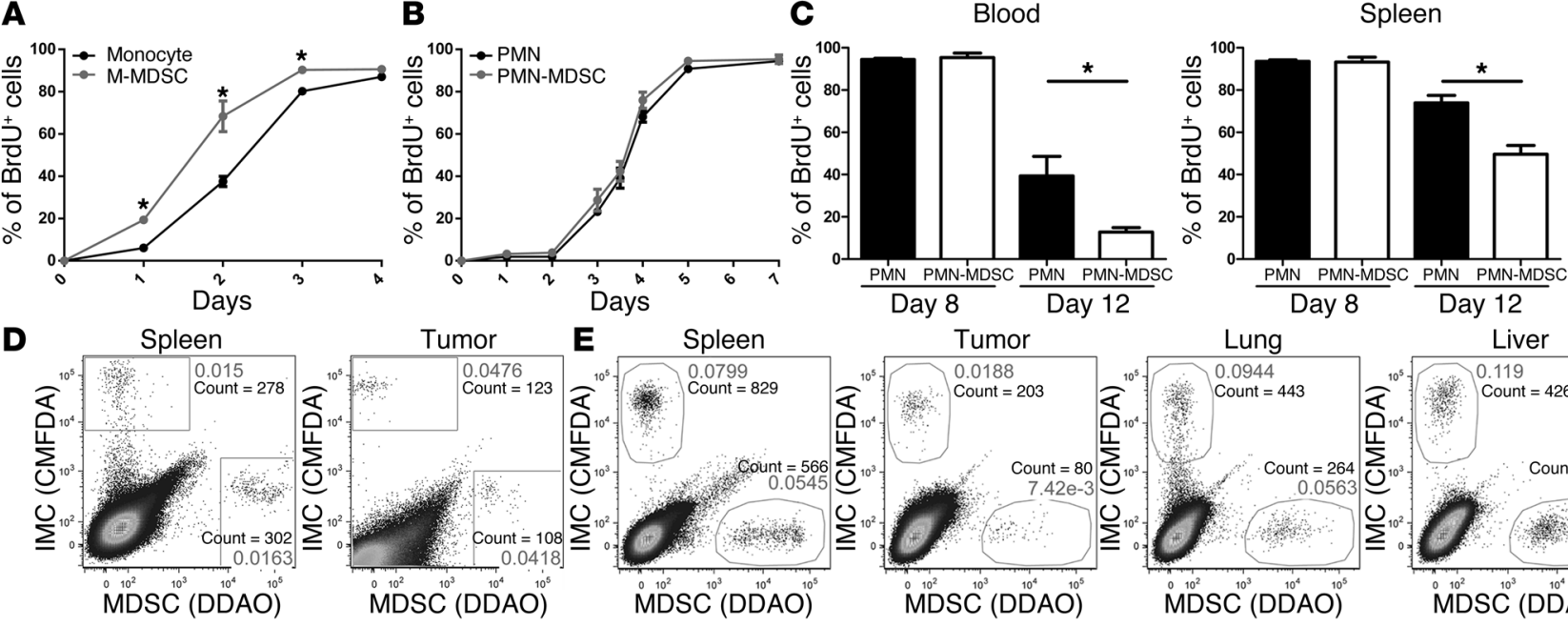

E

Spleen
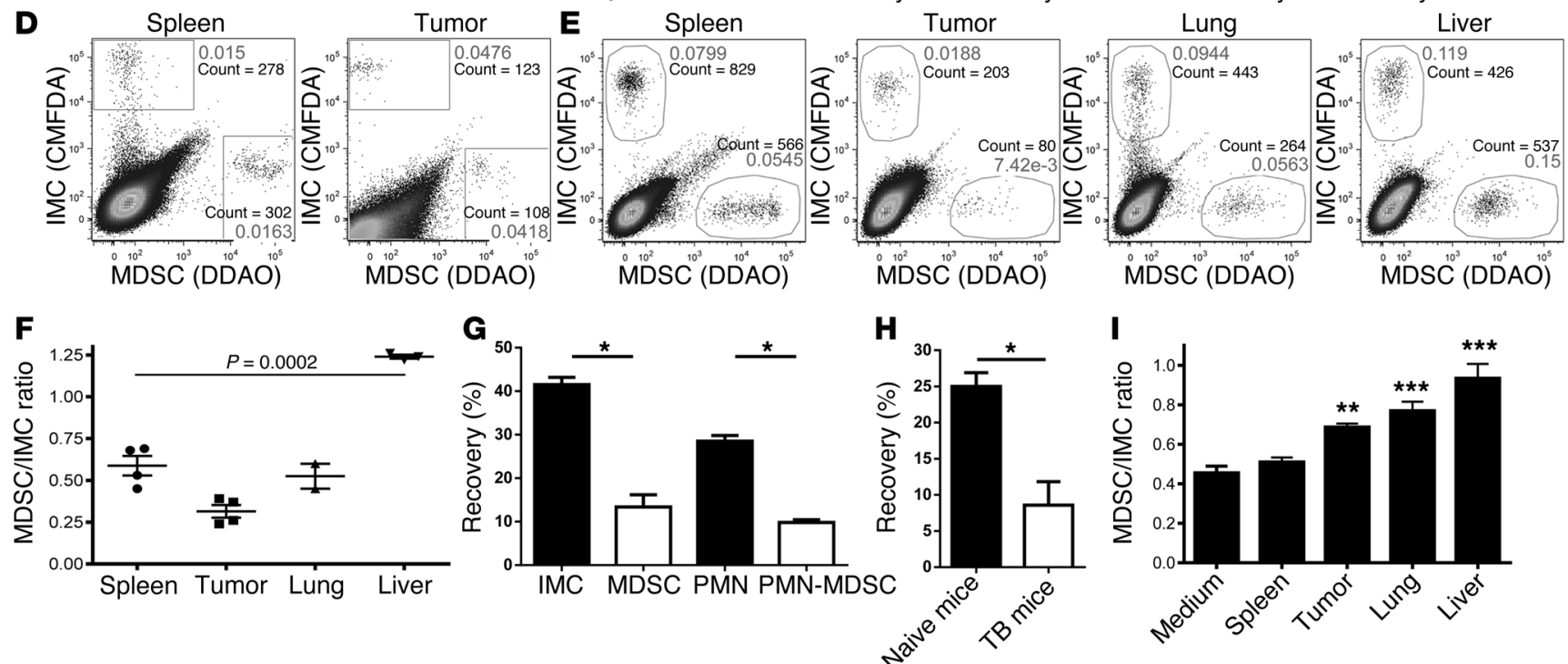

\section{Figure 1}

MDSCs have shorter survival than their naive counterparts. (A-C) BrdU pulse-chase analysis of MDSC turnover ( $n=3$ mice per time point). (A and $\mathbf{B})$ BrdU $^{+}$cells in PB monocytes and M-MDSCs (A) and in PB PMNs and PMN-MDSCs (B) during pulse. (C) BrdU ${ }^{+}$cells in PMNs and PMN-MDSCs in blood and spleen during chase. (D-F) MDSC/IMC ratio in different organs 6 (D) or 20 (E and F) hours after i.v. injection of MDSC/IMC mix (1:1 ratio) into TB recipients. ( $\mathbf{D}$ and $\mathbf{E}) 1$ representative experiment. Cell count and percentages of boxed regions are indicated. (F) Combined results; each symbol represents 1 mouse $(n=4)$. (G) Recovery of myeloid cells isolated from BM or spleen of control or EL4 TB mice, after 20 hours of culture in complete media without cytokines $(n=3) .2 \times 10^{5}$ purified cells were plated at the beginning of culture. (H) Naive and EL4 TB mice were injected i.p. with $12 \%$ casein 20 and 4 hours prior to harvesting of peritoneal fluid. Ly $6 G^{+}$cells were purified using magnetic beads and cultured overnight in complete media without cytokines. Shown is the proportion of surviving cells (relative to input) in 3 samples per group. (I) Purified IMCs and MDSCs were labeled with 2 different cell trackers (1:1 CMFDA/DDAO). Labeled cells were cultured alone in medium or mixed at a 1:5 ratio with freshly isolated splenocytes or with tumor, lung, or liver cells, then cultured overnight in complete media. The MDSC/IMC ratio was then calculated $(n=3)$. $P$ values are compared with medium alone. ${ }^{*} P<0.05 ;{ }^{* \star} P<0.01 ;{ }^{* \star \star} P<0.001$.

MDSCs had the same rate of BrdU uptake as PMNs (Figure 1B). In contrast to M-MDSCs, PMN-MDSCs do not proliferate (13), which explains the different kinetics of BrdU accumulation observed between M-MDSCs and PMN-MDSCs (Figure 1, $A$ and B). During the chase phase, we observed significantly accelerated loss of BrdU positivity by PMN-MDSCs compared with PMNs (Figure 1C). These differences were not due to different kinetics of replacement of labeled cells, since during the pulse phase, PMNs and PMN-MDSCs had similar rates of BrdU incorporation. Therefore, PMN-MDSCs either migrated to different organs or died faster than PMNs. To test these possibilities, we isolated the total population of $\mathrm{Gr} 1^{+} \mathrm{CD} 11 \mathrm{~b}^{+} \mathrm{MDSC}$ from $\mathrm{BM}$ of EL4 TB mice and immature myeloid cells (IMCs), with the same phenotype and purity, from naive mice as a control (Supplemental Figure 1B). MDSCs and IMCs were separately labeled with 2 fluorescent cell trackers (CMFDA and DDAO, mixed at a 1:1 ratio), and then injected i.v. into EL4 TB mice. At 6 hours after transfer, the MDSC/IMC ratio in spleens and tumors in the recip- ients remained the same (1:1; Figure 1D). However, when cells were evaluated 20 hours after transfer, the presence of MDSCs in spleen, tumor, and lung was lower than that of IMCs (Figure 1, $\mathrm{E}$ and F). The same results were obtained when the 2 cell trackers were switched between the cells (data not shown), which indicates that the observed differences were not the artifact of the cell labeling. The total number of cells evaluated in each organ was relatively small. However, the large differences in MDSC/IMC ratios between different tissues suggests that blood contamination was not directly responsible for those changes.

Next, we studied the proliferation and survival of these cells in vitro. In these experiments, in addition to the total population of BM-derived MDSCs, we also used spleen PMN-MDSCs and PMNs (Supplemental Figure 1C). Since the numbers of M-MDSCs and monocytes in spleen are low, these cells were not used in these experiments. The recovery of BM MDSCs and spleen PMN-MDSCs after overnight culture (without cytokines) was significantly lower than IMCs and PMNs from naive mice (Figure 1G). No differences 

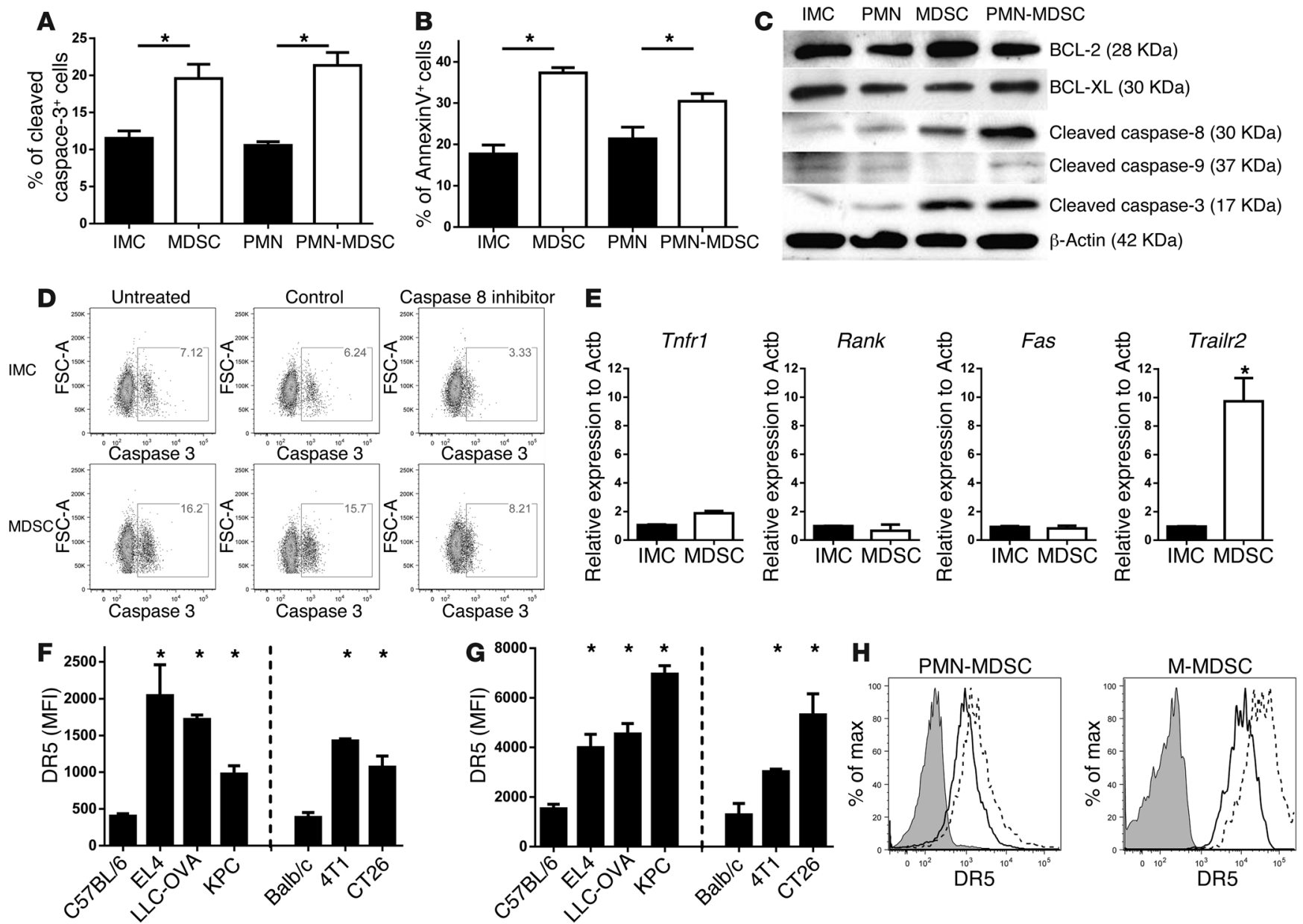

Figure 2

MDSCs undergo rapid apoptosis mediated by upregulation of DR5 expression. (A and B) Apoptosis was analyzed based on percentages of cleaved caspase- $3^{+}$cells after 3 hours in culture $(\mathbf{A})$ and of annexin $\mathrm{V}^{+}$cells after 6 hours $(\mathbf{B})$. Results represent the average of 4 different experiments. (C) Amount of BCL-2, BCL-XL, cleaved caspase-8, cleaved caspase-9, and cleaved caspase-3 was determined after a 3-hour culture of cells by Western blot. 3 experiments were performed with the same results. (D) Cells were cultured in the presence of the caspase-8 inhibitor Z-IETD-FMK (100 $\mu \mathrm{M})$ or DMSO (control), and the percentage of apoptotic cells (cleaved caspase-3+) was determined after 3 hours. Representative results of 3 different experiments are shown. (E) Receptor expression in BM IMCs or MDSCs, determined using quantitative RT-PCR. Results represent the average of 3 different samples. (F and $\mathbf{G})$ DR5 expression on the surface of PMN-MDSCs (F) or M-MDSCs $(\mathbf{G})$ in spleens of different TB mice or from control PMNs (F) or monocytes (G) isolated from corresponding C57BL/6 or Balb/c mice (3-5 different samples). (H) DR5 expression on the surface of PMN-MDSCs and M-MDSCs from spleen (solid line) or tumor site (dotted line) from EL4 TB mice. Gray filled histogram, isotype control. Results are representative of 4 different experiments. ${ }^{*}<0.05$.

in the low levels of proliferation were observed between MDSCs and IMCs (data not shown). To ascertain that the observed differences did not simply reflect the activated state of PMN-MDSCs, myeloid cells were mobilized to peritoneum of naive or EL4 TB mice with repeated i.p. injections of casein. The survival of $\mathrm{Ly}^{6} \mathrm{G}^{+}$ cells isolated from the peritoneal cavity of TB mice and cultured overnight was significantly lower than that of cells isolated from naive mice (Figure $1 \mathrm{H}$ ), which indicates that the observed differences in MDSC and IMC survival were not caused by differences in the activation state of the cells.

To assess the effect of the tissue microenvironment on cell survival, BM MDSCs and IMCs were mixed with cells obtained from different organs of TB mice and cultured overnight, and total cell number and MDSC/IMC ratio were evaluated. The low level of MDSC survival was not improved by the presence of splenocytes.
It was increased in the presence of tumor and lung cells, but still remained lower than IMCs. Only liver provided equal protection for MDSCs and IMCs (Figure 1I). Thus, these data indicated that in many tissues, MDSCs exhibit worse survival than IMCs.

$P M N-M D S C$ s rapidly undergo apoptosis mediated by DR5. Caspase-3 and annexin $V$ staining revealed that BM MDSCs and spleen PMN-MDSCs had significantly higher levels of apoptosis than BM IMCs and spleen PMNs, respectively (Figure 2, A and B). Increased apoptosis was not associated with changes in BCL-2, BCL-XL, or cleaved caspase-9, but with an increased amount of cleaved caspase-8 (Figure 2C). Inhibition of caspase- 8 with the specific inhibitor Z-IETD-FMK (14) substantially reduced apoptosis of MDSCs (Figure 2D), which suggests that this process was mediated by caspase- 8 and most likely involved the extrinsic death receptor pathway (15). To evaluate whether MDSCs are more prone to apoptosis 

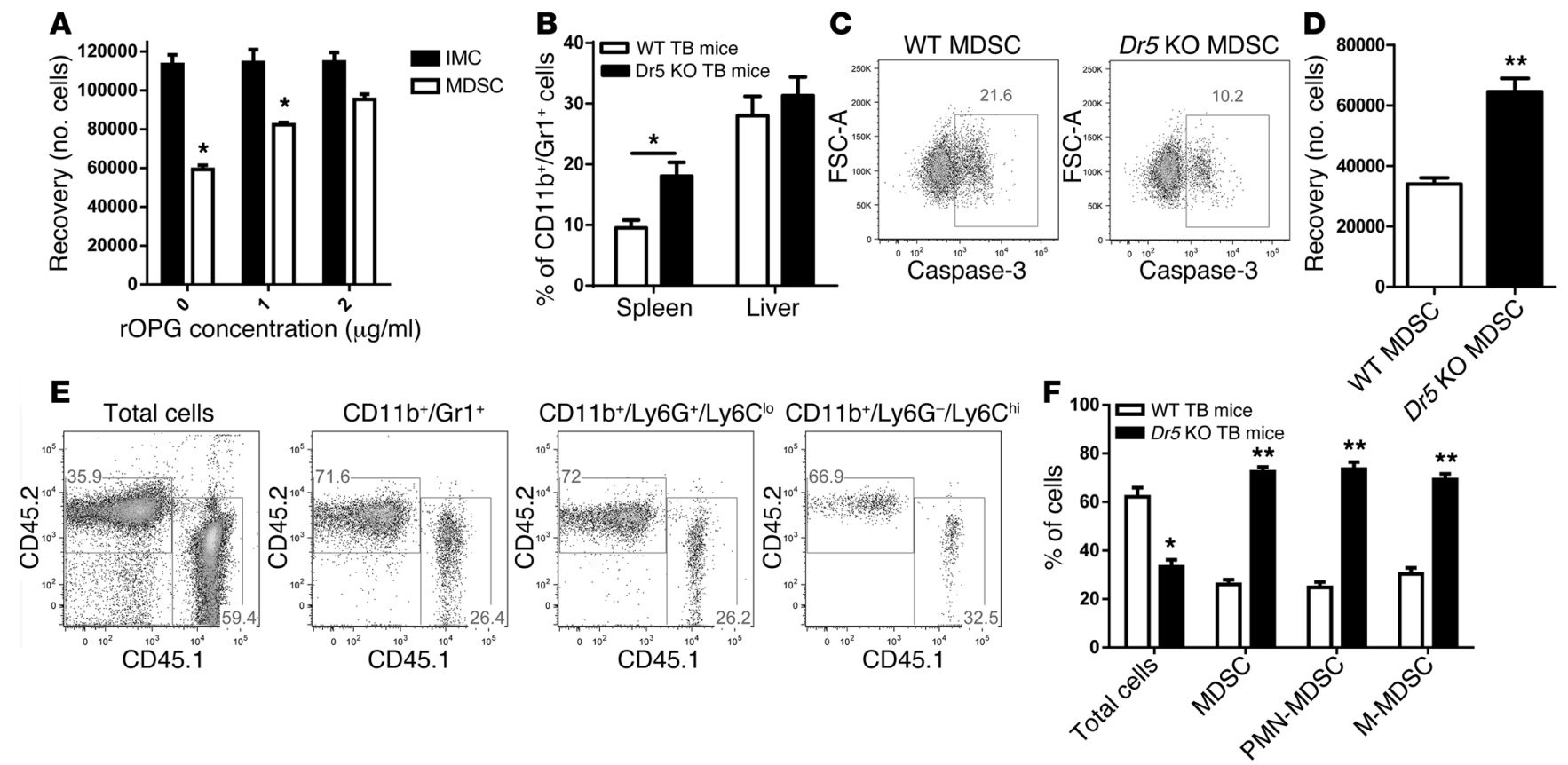

Figure 3

TRAIL-R is a potential target for MDSC removal. (A) IMCs and MDSCs $\left(2 \times 10^{5}\right.$ plated) were cultured in complete media overnight in the presence of different concentrations of recombinant OPG. Results represent the average number of cells recovered at the end of culture $(n=3)$. (B and C) Congenic CD45.1+ WT mice were lethally irradiated and reconstituted with WT or Dr5 KO BM cells. (B) 3 weeks after tumor inoculation, mice were sacrificed, and the presence of CD11b+Gr1+ MDSCs in spleen and liver was analyzed. (C) MDSCs were isolated from spleens and cultured in complete media. After 3 hours, the percentage of cleaved caspase- $3^{+}$cells was assessed by flow cytometry (results are representative of 5 experiments). (D) Number of cells recovered after overnight culture of $2 \times 10^{5}$ MDSCs (results of 5 different samples). (E and F) Naive CD45.1+ mice were lethally irradiated and reconstituted with a 1:1 mix of BM cells from CD45.2+ Dr5 KO and CD45.1+ WT mice ( $10^{6}$ cells). 5 weeks after reconstitution, mice were inoculated s.c. with EL4 cells, and spleens were collected 3 weeks later. The percentage of WT $\left(C D 45.1^{+}\right)$and Dr5 KO $\left(C D 45.2^{+}\right)$cells was analyzed in spleens among different cell populations. (E) Typical example of results. (F) Cumulative results of 3 experiments. ${ }^{*} P<0.05 ;{ }^{* \star} P<0.01$.

in vivo than their control counterparts, we used TUNEL staining in frozen spleen sections from EL4 TB and control mice. Very few $\mathrm{Gr}^{+}$cells in control spleens were TUNEL ${ }^{+}$, whereas the proportion of apoptotic cells among $\mathrm{Gr} 1^{+}$cells in $\mathrm{TB}$ mice was 5 times higher (Supplemental Figure 2).

To elucidate the mechanism of MDSC apoptosis, we analyzed the expression of known death receptors. No differences between MDSCs and IMCs were found in the expression of Tnfr1, Rank, or Fas, nor in the amount of FAS protein on the surface of the cells (Figure 2E and Supplemental Figure 3A). In contrast, expression of Trailr2 (also known as Tnfrsf $10 b$ and Dr5) was dramatically higher in MDSCs than in IMCs (Figure 2E). TNF-related apoptosis-induced ligand receptors (TRAIL-Rs) are members of the TNF receptor superfamily and consist of 2 death receptors, DR4 (also known as TRAIL-R1 and CD261; absent in mice) and DR5 (also known as TRAIL-R2 and CD262), 2 decoy receptors, DCR1 (also known as CD263) and DCR2 (also known as CD264), and 1 soluble receptor, osteoprotegerin (OPG) $(16,17)$. Spleen PMNMDSCs from mice bearing different transplantable tumors and spontaneous model of pancreatic carcinoma (KPC) had a significantly higher level of surface DR5 than corresponding PMNs from naive mice (Figure $2 \mathrm{~F}$ ). Similar results were obtained with M-MDSCs and monocytes (Figure 2G).

MDSCs present in tumor tissues of EL4 TB mice had substantially higher DR5 expression than MDSCs in spleens of the same mice (Figure 2H). Upregulation of DR5 was also observed in MDSCs obtained from the livers and lungs of TB mice (Supplemental Figure 3B). No differences in the amount of decoy TRAILRs were found between MDSCs and IMCs (Supplemental Figure 3, $\mathrm{C}$ and $\mathrm{D}$ ). We tested the possible effect of PMN activation on DR5 expression in casein-mobilized PMNs and PMN-MDSCs. PMNMDSCs in peritoneal fluid of casein-treated TB mice had significantly higher DR5 expression than did PMNs in naive mice analyzed under the same conditions (Supplemental Figure 3E).

Targeting of MDSCs using DR5. To determine whether TRAIL-Rs play a role in MDSC survival, we inhibited TRAIL signaling with OPG, a soluble decoy receptor for RANKL and TRAIL (18). Overnight culture of cells in the presence of OPG improved MDSC survival in a dose-dependent manner, but did not affect IMC survival (Figure 3A). To study the implication of TRAIL signaling on MDSC survival, we used myeloid cells from Dr5 KO mice $(19,20)$. Lack of DR5 did not affect differentiation of myeloid cells in vitro (data not shown). Reconstitution of the myeloid compartment remained unchanged between recipients reconstituted with BM from WT and Dr5 KO mice after lethal irradiation (data not shown). In mice reconstituted with Dr5 KO BM cells, EL4 tumors grew slightly faster than in mice reconstituted with WT BM cells $(P>0.1)$. However, lack of DR5 resulted in a significant increase in the proportion of MDSCs in TB mouse spleen $(P<0.05)$, but not liver (Figure 3B). Dr5 KO MDSCs had significantly lower apoptosis 

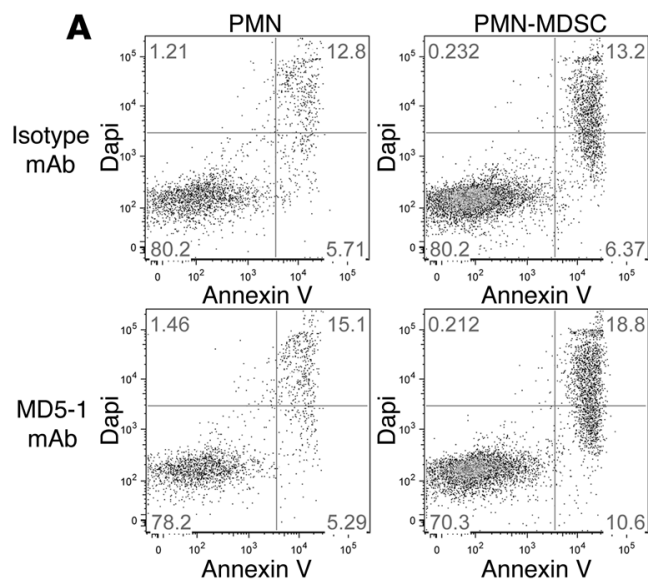

B

C
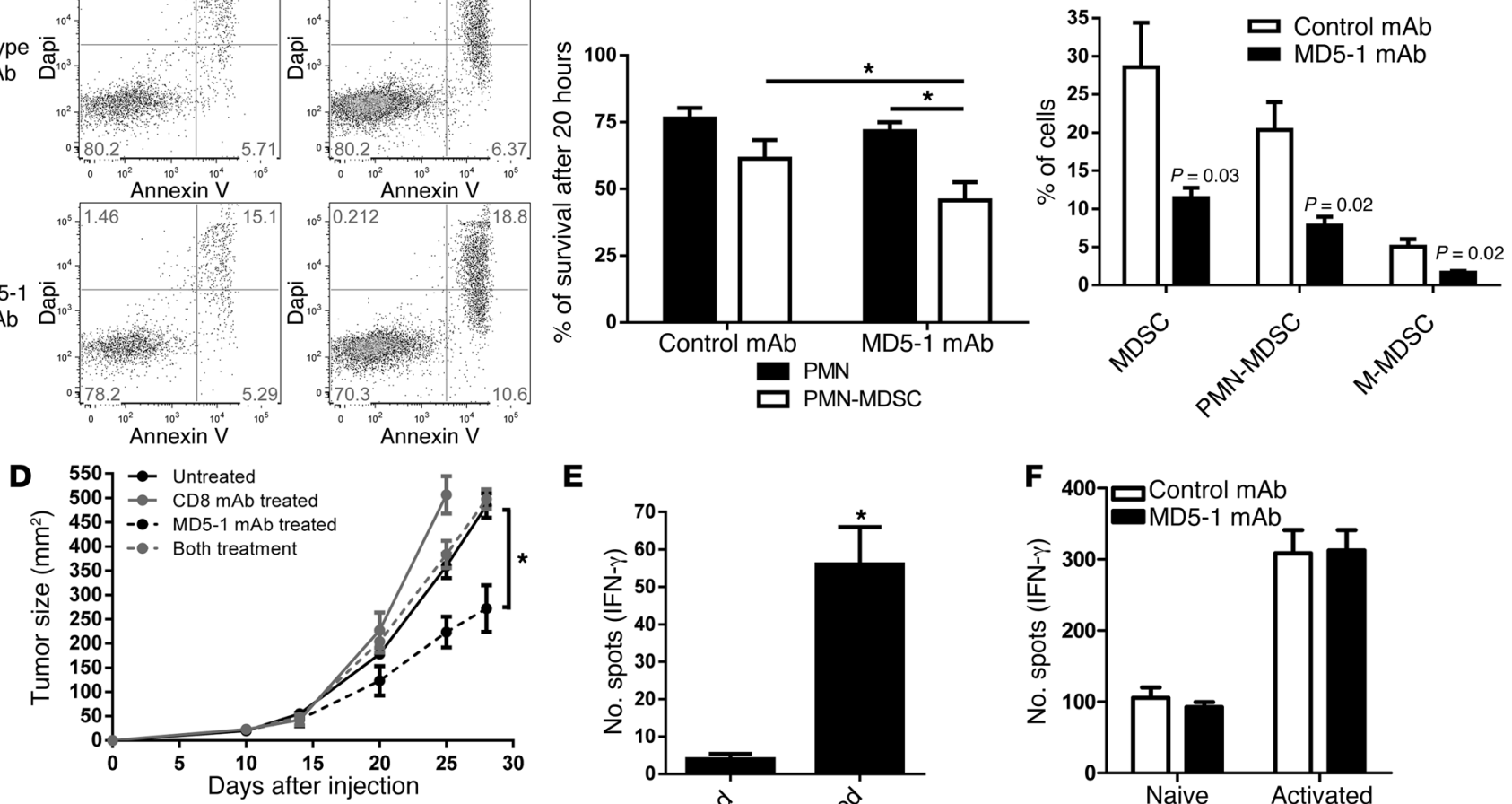

$\mathbf{E}$
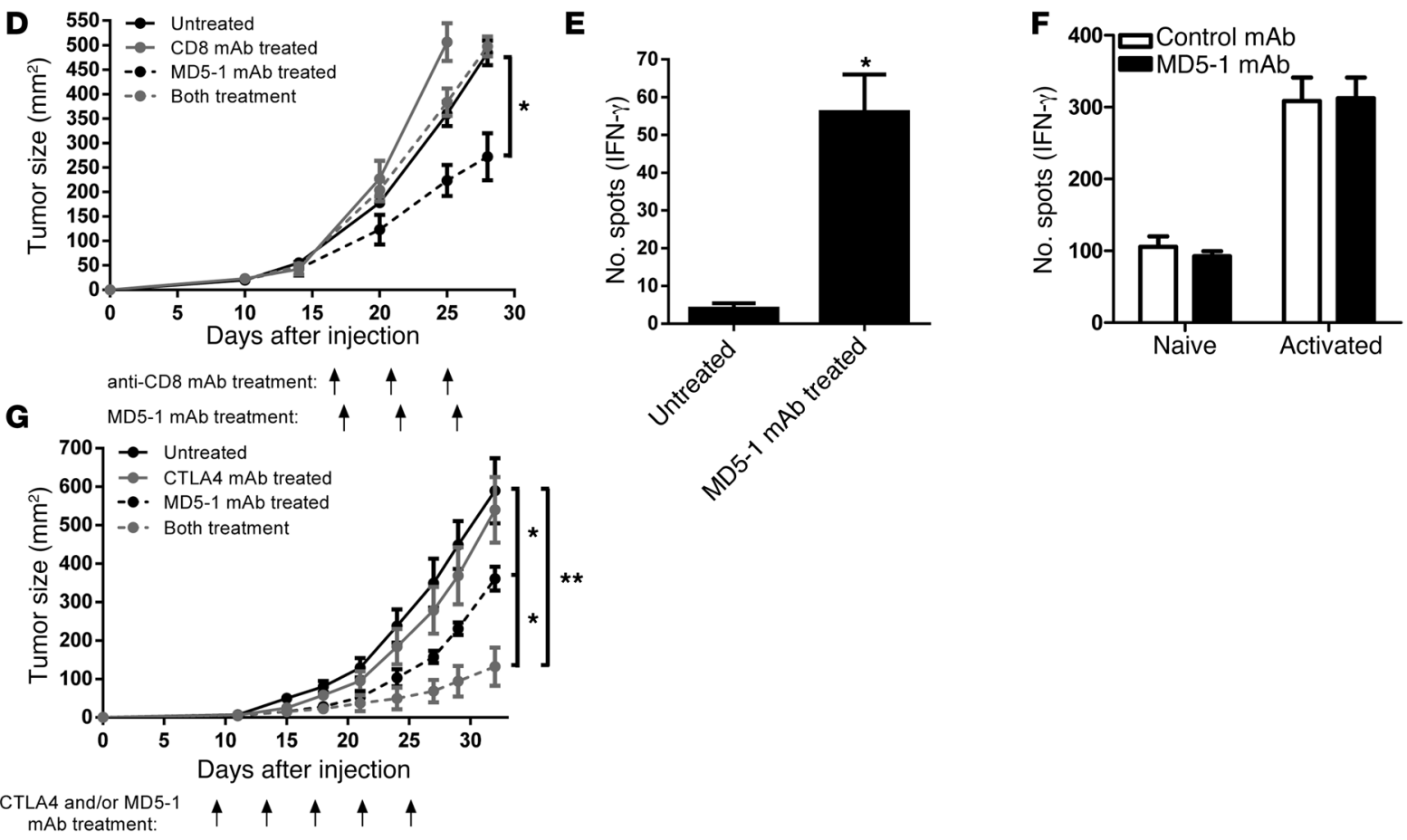

\section{Figure 4}

DR5 targeting results in selective MDSC elimination. (A and B) Splenic PMNs and PMN-MDSCs were cultured overnight in complete media supplemented with $10 \mathrm{ng} / \mathrm{ml} \mathrm{GM-CSF}$, in a plate coated with MD5-1 mAb or control lgG $(10 \mu \mathrm{g} / \mathrm{ml})$. After 20 hours, percentages of annexin $\mathrm{V}^{+}$cells (A) and survival (B) were determined. Results are representative of 3 different experiments. (C) Total MDSCs, PMN-MDSCs, and M-MDSCs in EL4 TB spleens, measured by flow cytometry. Treatment with control IgG and MD5-1 mAb $(100 \mu \mathrm{g})$ was initiated on day 17 after tumor inoculation, when tumor diameter reached $1.5 \mathrm{~cm}$, and repeated on days 20 and 23; mice were sacrificed on day 24. ( $n=4)$. (D) EG7 TB mice were treated with MD5-1 mAb (100 $\mu \mathrm{g})$ and/or anti-CD8 mAb (200 $\mu \mathrm{g})$ or left untreated, and tumor growth was determined ( $n=4$ per group). (E) On day 28 after tumor inoculation, mice were sacrificed; splenic T cells were enriched and stimulated in the presence of control DCs, loaded with OVA or irrelevant protein. IFN- $\gamma$ secretion was assessed by Elispot after 48 hours of restimulation $(n=3)$. (F) Naive (freshly isolated) or activated (3 days culture with $100 \mathrm{ng} / \mathrm{ml}$ SIINFEKL) OT-I splenocytes were stimulated with control or specific peptide (100 $\mathrm{ng} / \mathrm{ml} \mathrm{SIINFEKL)} \mathrm{in} \mathrm{the} \mathrm{presence} \mathrm{of} \mathrm{control} \mathrm{IgG}$ or MD5-1 mAb $(10 \mu \mathrm{g} / \mathrm{ml})$. IFN- $\gamma$ secretion was assessed by Elispot after 48 hours of restimulation $(n=3)$. (G) EL4 TB mice were treated with anti-CTLA4 and/or anti-MD5-1 mAb (100 $\mu$ g each) or left untreated, and tumor growth was determined $\left(n=4\right.$ per group). ${ }^{*} P<0.05 ;{ }^{* \star} P<0.01$.

after a 3-hour culture and significantly higher survival after overnight incubation than WT MDSCs (Figure 3, C and D), indicative of an important role of DR5 in MDSC survival. To verify these observations, we performed experiments using mixed BM chimera. Naive $\mathrm{CD} 45.1^{+}$mice were lethally irradiated and reconstituted with a 1:1 mix of BM cells from CD $45.2^{+}$Dr $5 \mathrm{KO}$ and CD $45.1^{+}$WT mice. Mice were inoculated with EL4 cells at 5 weeks after reconsti- tution, then analyzed 3 weeks later. CD45.1+ WT cells were prevalent in the total population of donor's cells in spleens (CD 45.1 $1^{+}$ WT, 60\%-70\%; CD 45.2+ Dr5 KO, 30\%-40\%). However, when the population of MDSCs and their subsets were evaluated, Dr5 KO cells represented the vast majority of the cells (>70\%; Figure 3, $\mathrm{E}$ and F). These results strongly suggested that Dr5 KO MDSCs had a survival advantage over WT MDSCs. 


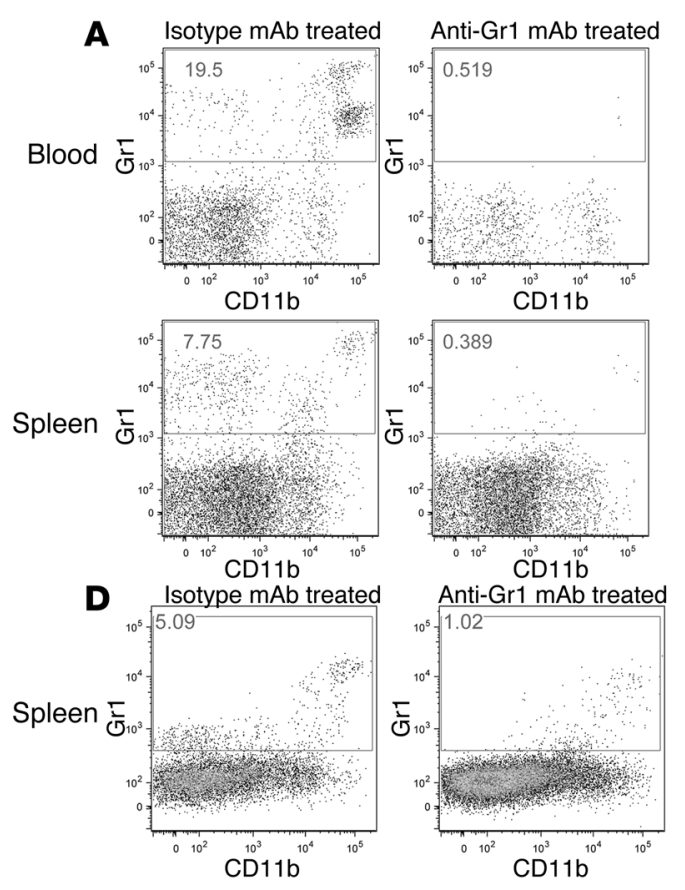

B C
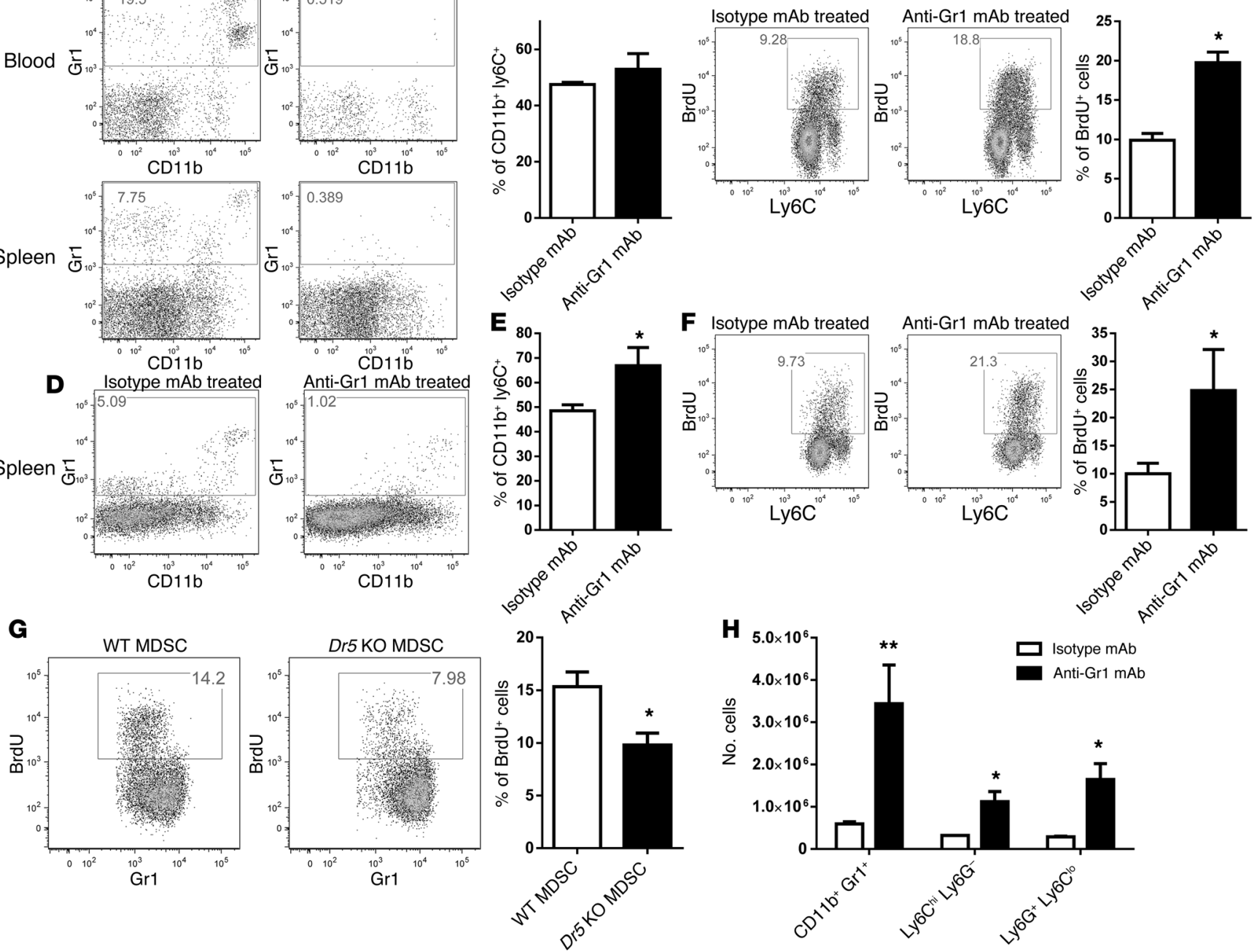

Figure 5

Short life-span of MDSCs contributes to their expansion. (A-F) Naive mice received 1 injection of $250 \mu \mathrm{g}$ anti-Gr1 mAb to deplete PMNs from the periphery, then examined $2(\mathbf{A}-\mathbf{C})$ and $4(\mathbf{D}-\mathbf{F})$ days after treatment. (A and $\mathbf{D})$ Gr1 depletion was assessed in blood and spleen; typical results are shown. (B, C, E, and F) Mice were then injected with BrdU i.p. and sacrificed 4 hours later. (B and E) BM IMCs, assessed by flow cytometry $(n=3)$. (C and F) BrdU incorporation in BM IMCs (CD11b+Ly6C+ cells gated), assessed by flow cytometry. Shown are 1 representative sample (left) and cumulative results (right; $n=3$ ). (G) 3 weeks after EL4 inoculation, TB WT or Dr5 KO mice were injected i.p. with BrdU for 4 hours, and spleen MDSCs were analyzed for BrdU incorporation (percent BrdU+ cells). Shown are 1 representative sample (left) and cumulative results (right; $n=3$ ). (H) Mice were injected i.p. with $250 \mu \mathrm{g} \mathrm{Gr} 1$ or control IgG mAb. The absolute number of monocytes and PMNs was determined in spleens 9 days after injection $(n=3)$. ${ }^{\star} P<0.05 ;{ }^{* \star} P<0.01$.

Our data suggested that high DR5 expression might be a unique characteristic of MDSCs. Thus, we tested the possibility of selective targeting MDSCs using an agonistic DR5 antibody, MD5-1 mAb. Myeloid cells were cultured in the presence of GM-CSF to support their survival. Treatment with MD5-1 mAb caused apoptosis of PMN-MDSCs, but not PMNs, which resulted in a significant decrease in PMN-MDSC survival $(P<0.05)$, but not PMN survival, after a 20-hour culture (Figure 4, A and B).

To test the effect of MD5-1 mAb in vivo, we used EL4 cells and their OVA-expressing derivate (EG7 cells, which are resistant to MD5-1 mAb) in contrast to 4T1 mammary carcinoma, an example of DR5-sensitive tumor (Supplemental Figure 4). In mice bearing large EL4 tumors (1.5 cm in diameter), treatment with
3 injections of MD5-1 mAb strongly decreased the accumulation of both MDSC subsets (Figure 4C). The effect of MD5-1 mAb was quite specific for MDSCs, since the presence of other populations of myeloid cells (DCs and macrophages) was not changed, and the proportion of $\mathrm{T}$ and $\mathrm{B}$ cells was increased (likely due to the effect of MDSC depletion) (Supplemental Figure 5). Treatment with MD5-1 mAb alone was able to significantly delay tumor progression in mice bearing immunogenic EG7 tumor $(P<0.05$; Figure 4D). The antitumor effect was mediated by $\mathrm{CD}^{+} \mathrm{T}$ cells, since it was abrogated by the administration of CD8 antibody (Figure 4D), and MD5-1 mAb treatment resulted in a substantial increase in the number of OVA-specific CD8 $8^{+} \mathrm{T}$ cells $(\sim 10$-fold; Figure 4E). Since activated T cells express DR5 $(21,22)$, we tested 


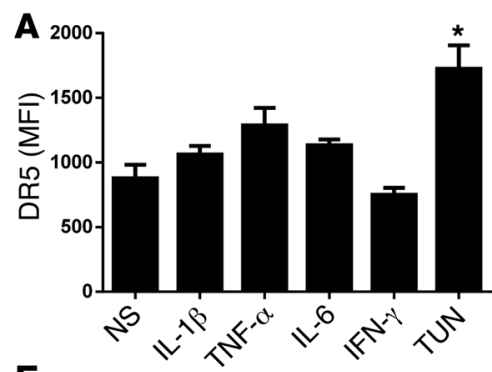

E
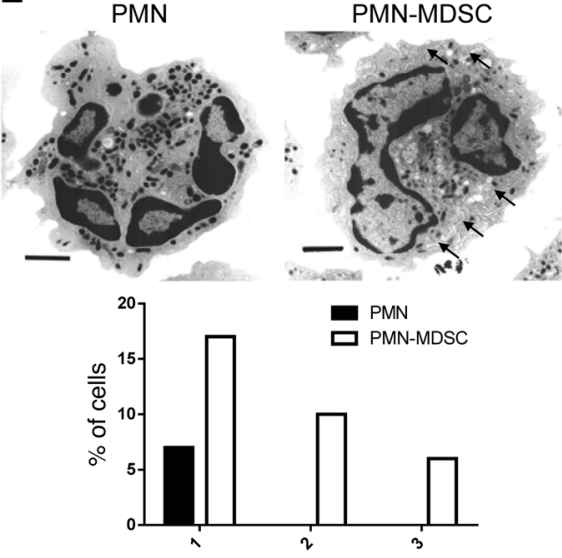

ER dilation score

H

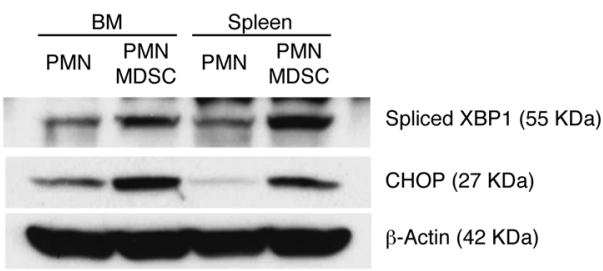

$\mathbf{F}$
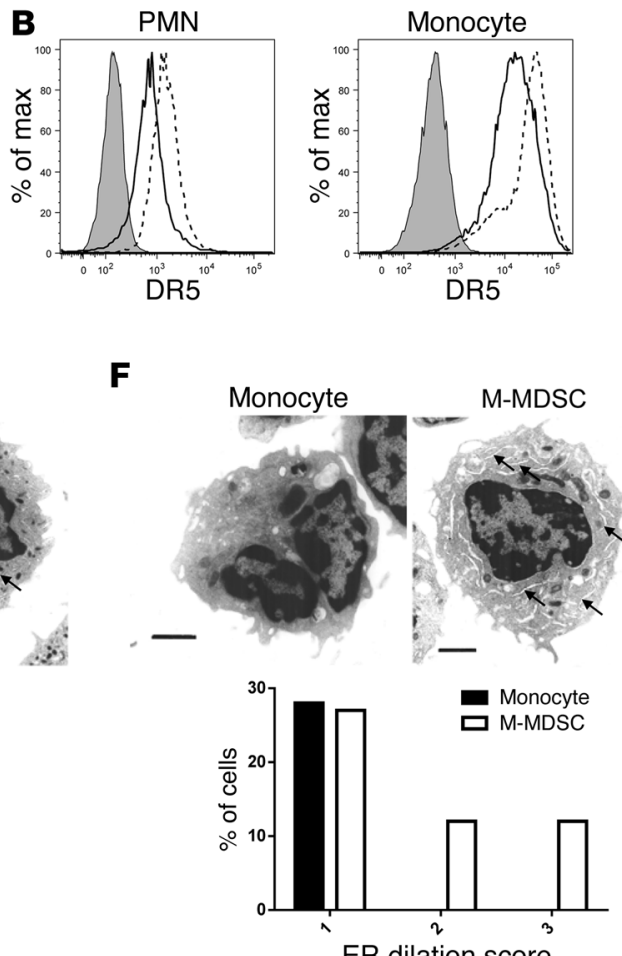

ER dilation score

\section{I}

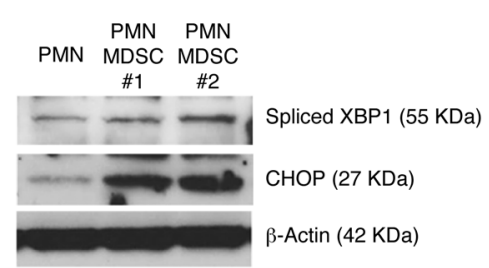

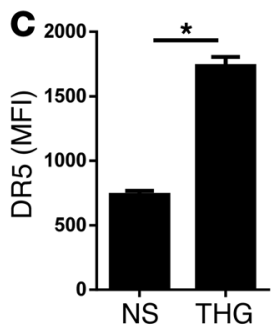

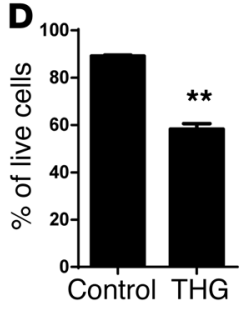

G
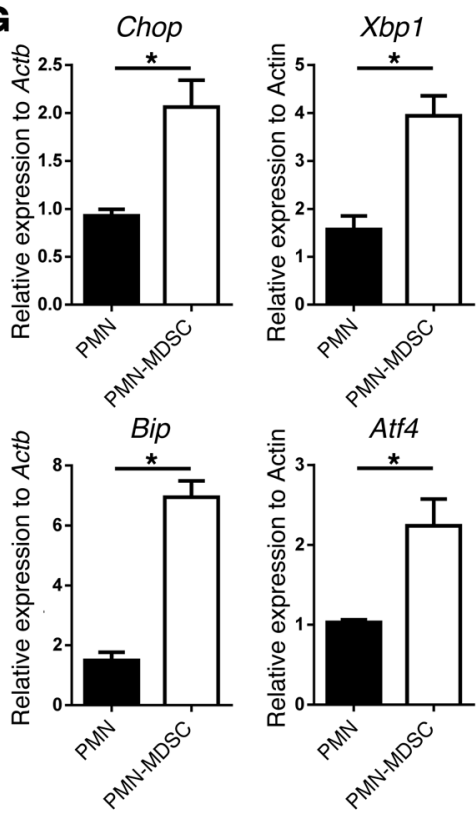

Figure 6

ER stress response drives TRAIL-R upregulation in MDSCs. (A and B) Hematopoietic progenitor cells were cultured for 5 days in the presence of $20 \mathrm{ng} / \mathrm{ml} \mathrm{GM-CSF}$. (A) DR5 expression on the surface of PMNs stimulated for 24 hours in the presence of IL-1 $\beta$, TNF- $\alpha$, IL-6, IFN- $\gamma$, or tunicamycin (TUN) or left unstimulated (NS). Results of 3 experiments are shown. (B) DR5 expression on the surface of PMNs and monocytes stimulated with tunicamycin (dotted line) or left unstimulated (solid line) for 24 hours. Gray filled histogram, isotype control. Shown is 1 representative experiment of 4. (C and D) Purified PMNs from BM were treated overnight with thapsigargin (THG) or left untreated. DR5 expression (C) and cell viability (D) were assessed by flow cytometry $(n=3)$. ( $\mathbf{E}$ and $\mathbf{F})$ Electron microscopy analysis of cell morphology. PMNs and PMNMDSCs (E) and monocytes and M-MDSCs (F) were isolated from spleen of control and EL4 TB mice. Shown are representative microphotographs (arrows denote ER dilation) and the proportion of cells with each ER dilation score (see Methods). Scale bars: $1 \mu \mathrm{m}$. (G) Chop, Xbp1, Atf4, and Bip expression in splenic PMNs and PMN-MDSCs, determined using quantitative RT-PCR. Results are from 3 different samples. (H and I) Spliced XBP1 and CHOP in PMNs and PMN-MDSCs from EL4 TB mice (H) or KPC TB mice (spleen cells only; I), determined by Western blot. Results are representative of 4 different samples. ${ }^{\star} P<0.05 ;{ }^{* \star} P<0.01$.

the possibility that triggering of the receptor could result in activation of T cells. No direct effect of MD5-1 mAb on the response of transgenic OT-1 T cells was observed (Figure 4F).

CTLA4 antibody has been used to enhance the generation of tumor-reactive $\mathrm{CD}^{+} \mathrm{T}$ cells (23). By itself, this antibody had no significant effect on the growth of established poorly immunogenic EL4 tumor (Figure 4G). However, the combination of CTLA4 and MD5-1 antibodies resulted in a remarkable retardation of tumor growth $(P<0.01$; Figure $4 \mathrm{G})$. These results indicate that elimination of MDSCs via targeting of DR5 in combination with CTLA4 blockade results in a potent antitumor effect.

Short lifespan of MDSCs can contribute to their expansion and accumulation. Since it is known that lymphodepletion can induce homeo- static proliferation of lymphocytes (24), we hypothesized that the reduced survival of MDSCs in the periphery could contribute to increased production of these cells in the BM. To model this situation in tumor-free mice, we used Gr1-specific mAb, which effectively depleted $\mathrm{Gr} 1^{+}$PMNs and monocytes in $\mathrm{PB}$ and spleens 48 hours after its administration, but had little effect on the cells in BM (Figure 5, A and B). At that time, BM IMCs from Gr1 mAbtreated mice had higher proliferation than cells from untreated control mice (Figure 5C). 2 days later, PMNs and monocytes remained depleted in the periphery, but IMCs significantly accumulated in BM (Figure 5, D and E). The proliferation of BM IMCs remained significantly increased in mice depleted of $\mathrm{Gr}^{+}$cells in the periphery (Figure 5F). Dr5 KO MDSCs, which had longer 



$\mathbf{F}$

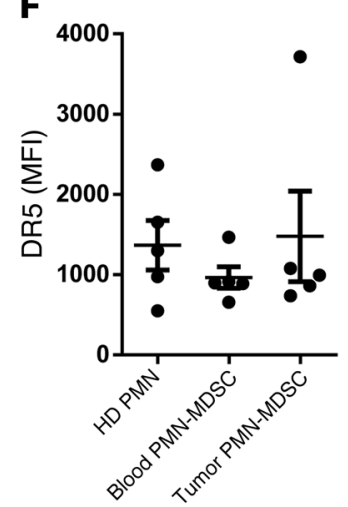

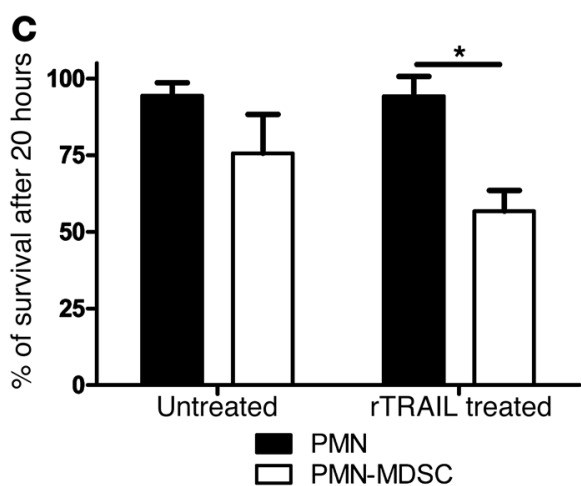
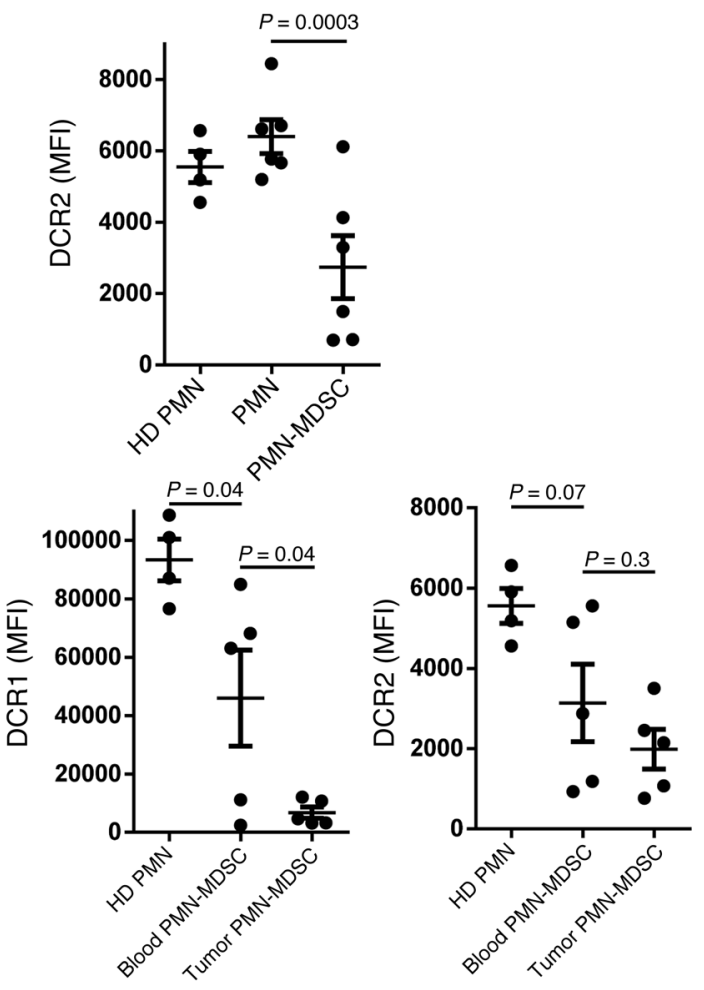

Figure 7

Human MDSC survival and TRAIL-R expression. (A) Human PMN-MDSCs and PMNs were isolated from blood of NSCLC patients and cultured overnight in complete media. The percentage of cells was determined by flow cytometry at the beginning and end of culture, and the total number of surviving PMN-MDSCs and PMNs was calculated $(n=3)$. (B and C) Human PMN-MDSCs and PMNs were cultured in complete media supplemented with $10 \mathrm{ng} / \mathrm{ml} \mathrm{GM}-\mathrm{CSF}$, in the presence of agonistic anti-DR5 mAb (200 ng/ml) (B) or recombinant TRAIL (100 $\mathrm{ng} / \mathrm{ml})(\mathbf{C})$, and the percentage of surviving cells was determined $(n=3)$. (D) DR4, DR5, DCR1, and DCR2 expression on the surface of PMN-MDSCs and PMNs from the blood of healthy donors (HD) or NSCLC patients. (E) DCR1 and DCR2 expression in PMNs (solid line) and PMN-MDSCs (dotted line). Gray filled histogram, isotype control. Results show a typical example of 6 different patient samples. (F) DR5, DCR1, and DCR2 expression on the surface of blood or tumor PMN-MDSCs from 5 HNC patients and PMNs from healthy donors. ${ }^{*} P<0.05 ;{ }^{* *} P<0.01$.

lifespan than WT MDSCs, also had significantly lower proliferation than WT MDSCs (Figure 5G). We then sought to determine whether myeloid cell accumulation would be observed in spleen when the effect of antibody in periphery had stopped. 9 days after treatment with Gr1 mAb, mice had splenomegaly and more than doubled total splenocyte numbers compared with control IgGtreated controls (Supplemental Figure 6, A and B). The proportion and absolute number of both monocytes and PMNs were significantly higher in spleen of Gr1 mAb-treated mice than in control spleens (Supplemental Figure 5H and Supplemental Fig- ure $6 \mathrm{C})$. Together, these data suggest that the shortened lifespan of MDSCs in the periphery can stimulate the proliferation of their precursors in BM, supporting their expansion in TB hosts.

Mechanism of the regulation of DR5 expression in MDSCs. DR5 expression in tumor cells can be induced by NF- $\kappa \mathrm{B}$, which is regulated by proinflammatory cytokines (25). We asked whether increased DR5 expression in MDSCs could be mediated by various proinflammatory cytokines present in tumor milieu. Treatment of BM IMCs from naive mice with IL- $1 \beta$, IL- 6 , TNF- $\alpha$, or IFN- $\gamma$ in the absence or presence of tumor explant supernatants (TES) failed 
A
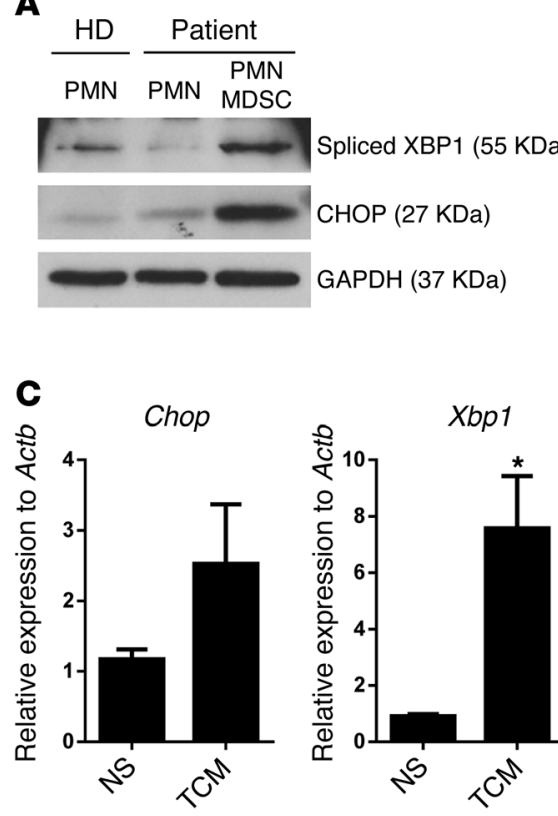

B
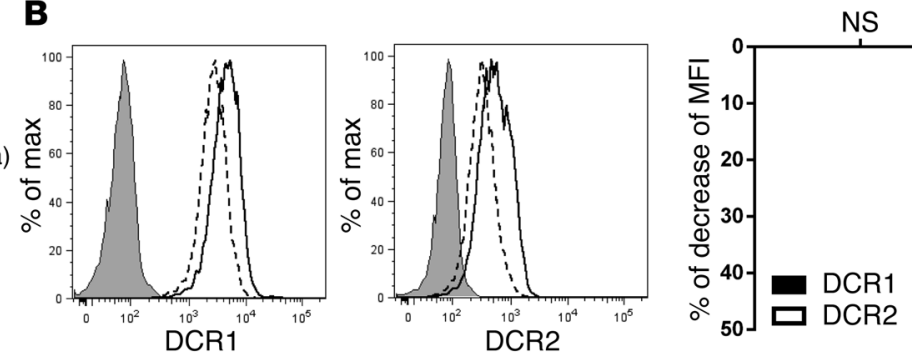

D
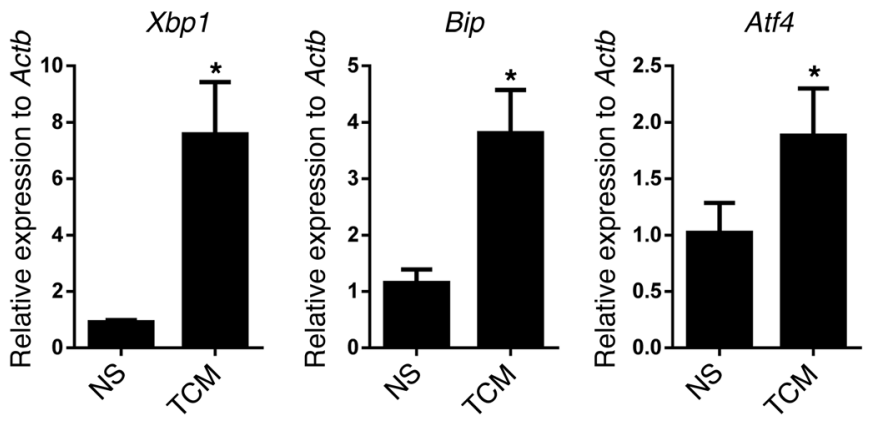

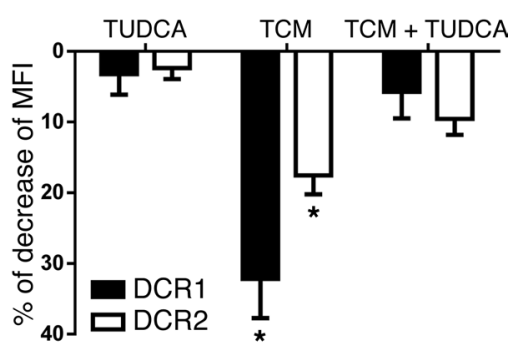

Figure 8

ER stress response modulates TRAIL-R expression in human MDSCs. (A) Amounts of spliced XBP1 and CHOP in PMNs and PMN-MDSCs purified from healthy donor or NSCLC patient blood, determined by Western blot. Results are representative of 5 different samples. (B) Human PMNs from healthy donors were cultured in complete media supplemented with $10 \mathrm{ng} / \mathrm{ml}$ GM-CSF for 24 hours in the presence of $1 \mu \mathrm{M}$ thapsigargin. DCR1 and DCR2 expression was assessed by flow cytometry. Shown are representative flow histograms and percentage of MFI decrease $(n=3)$. (C and D) Healthy donor PMNs were cultured with GM-CSF for 48 hours in the presence of $20 \%$ TCM (PCI30 cells). (C) Chop, Xbp1, Atf4, and Bip expression, determined by quantitative RT-PCR. Results represent the average of 4 different samples (D) PMNs were preincubated with $500 \mu \mathrm{g} / \mathrm{ml}$ TUDCA (Calbiochem) for 3 hours prior to the addition of TCM. After culture, cells were harvested, and DCR1 and DCR2 expression was analyzed. Data are mean \pm SD of 4 different samples. ${ }^{*} P<0.05$.

to upregulate DR5 expression (Figure 6A and data not shown). We confirmed that, at selected concentrations, those cytokines activated their respective signaling pathways: NF- $\mathrm{\kappa B}, \mathrm{STAT} 1$, and STAT3 (Supplemental Figure 7). DR5 expression in tumor cells can also be induced by ER stress via spliced XBP1, a major mediator of the IRE1 pathway, and C/EBP homologous protein (CHOP), a downstream molecule of the PERK pathway (24, 26-29). The ER stress inducer tunicamycin caused significantly increased DR5 expression in BM PMNs and monocytes (Figure 6, A and B). We confirmed these results by treating BM PMNs with thapsigargin, another ER stress inducer (Figure 6C). Upregulation of DR5 was associated with significantly decreased PMN survival (Figure 6D). Thapsigargin did not affect the expression of decoy TRAIL-Rs (Supplemental Figure 8A). Consistent with lack of the effect on DR5 expression, TES alone did not induce the ER stress response in murine PMNs (Supplemental Figure 8B).

To evaluate the possible biological significance of these findings, we examined whether signs of ER stress could be found in MDSCs directly isolated from TB mice. One of the hallmarks of ER stress is enlargement of the ER (30). Using electron microscopy, we observed an increased percentage of cells with dilated ER in spleen PMN-MDSCs compared with PMNs from EL4 TB mice (Figure 6E). Similar results were observed in M-MDSCs compared with monocytes (Figure 6F). Expression of several ER stress response pathway markers - Chop, Xbp1, Bip, and Atf4was significantly upregulated in PMN-MDSCs compared with PMNs (Figure 6G). The amounts of these proteins in spleen
PMN-MDSCs from EL4 TB mice and from mice with spontaneous pancreatic cancer were higher than in PMNs from naive or WT mice (Figure 6, H and I). We also tested the possible effect of PMN activation on the ER stress response in caseinmobilized PMNs and PMN-MDSCs. PMN-MDSCs in peritoneal fluid of casein-treated TB mice had substantially higher spliced XBP1 and CHOP expression than did PMNs analyzed under the same conditions in naive mice (Supplemental Figure 8C). Thus, MDSCs exhibited clear signs of the ER stress response.

Human MDSCs are sensitive to TRAIL-induced cell death. To assess the survival of MDSCs in cancer patients, we isolated the population of $\mathrm{CD} 11 \mathrm{~b}^{+} \mathrm{CD} 33^{+} \mathrm{CD} 14^{-} \mathrm{CD} 15^{+} \mathrm{PMN}-\mathrm{MDSC}$ from the mononuclear fraction of $\mathrm{PB}$ of patients with non-small cell lung cancer (NSCLC) (Supplemental Figure 9). These cells represent the most abundant group of MDSCs in patients with many types of cancer $(31,32)$. PMNs, with the same phenotype, were purified from $\mathrm{PB}$ of the same patients and from healthy donors using the standard neutrophil isolation protocol (33). PMNs survived 20 hours in culture without cytokines significantly better than did PMN-MDSCs $(P<0.01$; Figure 7A). Targeting TRAIL-Rs with either human DR5 agonistic antibody or recombinant TRAIL did not affect PMN survival, but significantly reduced PMN-MDSC survival (Figure 7, B and C).

We then analyzed TRAIL-R expression and found that, in contrast to mice, PMNs and PMN-MDSCs did not differ in the expression of surface DR4 or DR5 (Figure 7D). However, PMN-MDSCs from NSCLC patients had markedly lower expression of decoy recep- 
A
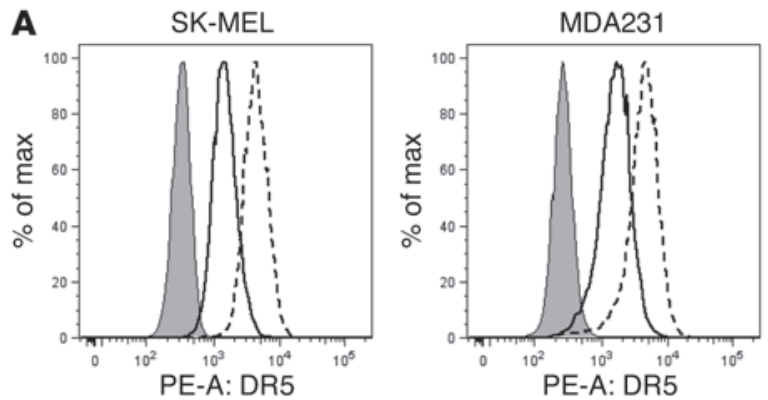

B

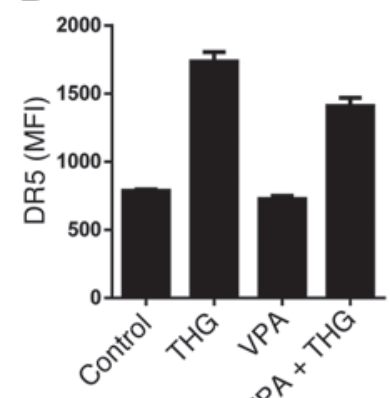

C

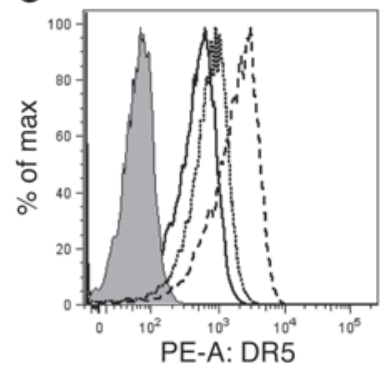

D

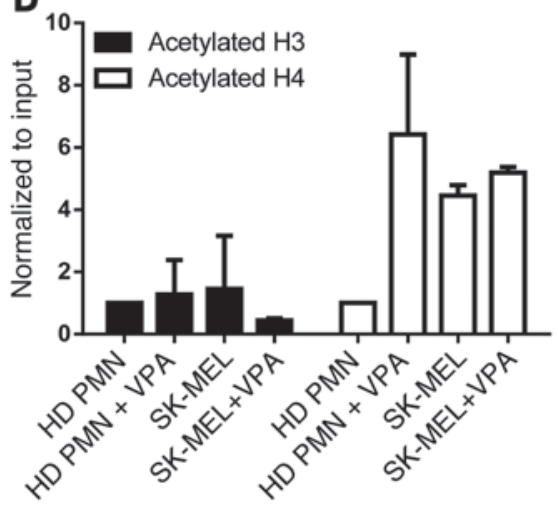

E

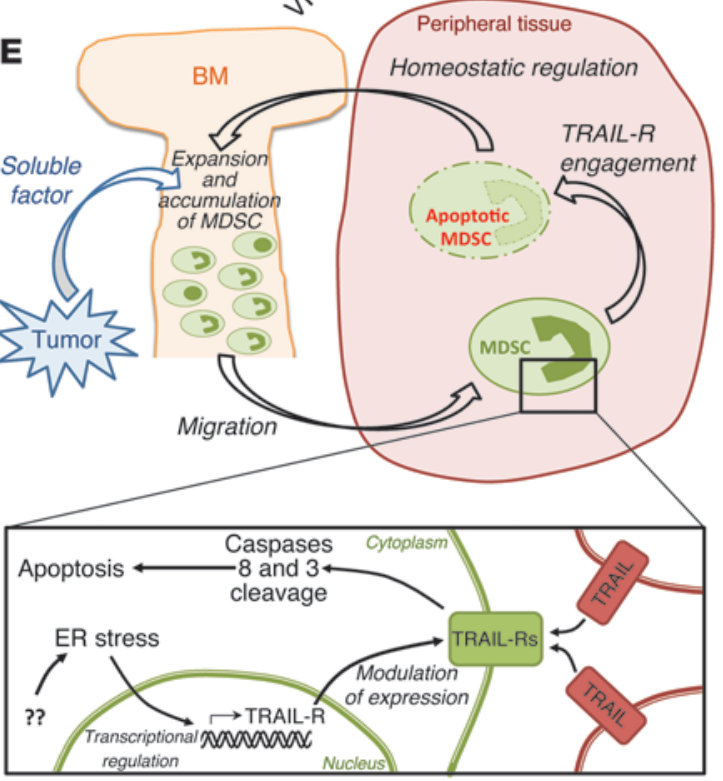

Figure 9

Mechanism of different regulation of DR5 in human and mouse PMNs. (A) SK-MEL melanoma tumor cells and MDA231 breast cancer cells were treated overnight with thapsigargin (dotted line) or left untreated (solid line), and DR5 expression was assessed by flow cytometry. Gray filled histogram, isotype control. Typical result is shown. (B) Mouse PMNs were purified from BM of naive mice and cultured for 24 hours in the presence of thapsigargin in combination with VPA. DR5 expression was assessed by flow cytometry $(n=3)$. (C) Effect of VPA and THG combination on DR5 expression in PMNs. DR5 expression was assessed by flow cytometry. Gray filled histogram, isotype control; black solid line, untreated; gray solid line, VPA alone; dotted line, VPA plus THG. Shown is 1 representative staining of 3 different experiments. (D) ChIP of $D R 5$ promoter with acetylated histone $\mathrm{H} 3$ or acetylated histone $\mathrm{H} 4$ antibodies in healthy donor PMNs and the SK-MEL melanoma cell line cultured overnight with or without VPA. Results are expressed as DNA enrichment normalized to corresponding input value. (E) Potential role of ER stress and TRAIL-Rs in the fate of MDSCs.

tors DCR1 and DCR2 (Figure 7, D and E). To verify and extend these findings, we evaluated samples from patients with head and neck cancer (HNC). TRAIL-R expression on MDSCs from PB and tumors was compared in the same patients. Although no difference in DR5 expression was seen, DCR1 expression was significantly lower in PB PMN-MDSCs than PB PMNs from healthy donors (Figure 7F). PMN-MDSCs from tumor site had markedly lower DCR1 expression than PB PMN-MDSCs in the same patients, and a similar trend was seen for DCR2 expression (Figure 7F).

ER stress response is activated in buman MDSCs and modulates decoy TRAIL-Rs. Next, we tested whether ER stress response was activated in human MDSCs. PMN-MDSCs from NSCLC patients exhibited strong upregulation of spliced XBP1 and CHOP protein expression compared with PMNs from the same patients or from healthy donors (Figure 8A). To test the possibility that the ER stress response could directly affect DCR expression, we treated PMNs from healthy donors with thapsigargin and observed decreased DCR1 and DCR2 expression, but no change in DR5 (Figure 8B and data not shown). A 48-hour incubation of PMNs from healthy donors with head and neck tumor cell (PCI30) conditioned medium (TCM) in the presence of GM-CSF induced marked upregulation of the ER stress response in these cells and caused significant downregulation of DCR1 and DCR2 (Figure 8, C and D). To test the possibility that the effect of TCM on TRAIL-R decoy expression was caused by ER stress, we used the well-known ER stress inhibitor TUDCA (tauroursodeoxycholic acid, sodium salt; ref. 34). TUDCA completely abrogated TCM-inducible downregulation of DCR1 and DCR2 in PMNs (Figure 8D).

ER stress inducers upregulated DR5 expression in human tumor cells (Figure 9A). We asked why this effect was not observed in human PMNs. Deacetylation of the DR5 promoter could regulate the expression of the receptor. We treated mouse PMNs (in 
which ER stress inducers were able to upregulate DR5 expression) with the pan-HDAC inhibitor valproic acid (VPA), alone or in combination with thapsigargin. In these experiments, VPA did not modulate the effect of thapsigargin on DR5 (Figure 9B). However, in human PMNs, treatment with VPA restored the ability of thapsigargin to induce DR5 expression (Figure 9C). To verify the DR5 promoter acetylation in human PMNs, we performed a ChIP assay. Treatment of tumor cells (as a control) with VPA did not affect the already high level of acetylated histone in the DR5 promoter. In contrast, VPA dramatically increased its level in PMNs (Figure 9D). These results indicate that DR5 promoter silencing by HDACs in human PMNs may prevent the upregulation of DR5 expression.

\section{Discussion}

Here, we present evidence that accumulation of MDSCs in TB mice and in cancer patients is not associated with increased survival of these cells. On the contrary, MDSCs had substantially shorter lifespan than PMNs and monocytes. This was evident in spleen, lung, and tumor tissues. In contrast, survival of MDSCs in liver was indistinguishable from that of IMCs, and DR5 deficiency did not affect MDSC accumulation in the liver. These data are consistent with several observations demonstrating that the liver is a site of large MDSC accumulation in TB mice and chronic infections $(35,36)$. Spleen is one of the major sites of MDSC expansion in TB mice. Our results indicative of a hostile spleen environment for MDSCs are surprising. However, the fact that spleen is a major source of extramedullar myelopoiesis in TB mice and the data on high monocyte turnover in spleens $(37,38)$ may suggest that MDSCs rapidly leave spleen and are being replaced with newly produced cells. Short survival of MDSCs in TB mice can stimulate proliferation of their precursors and further trigger their expansion (Figure 9E). We modeled this situation in tumor-free mice by using Gr1 mAb in vivo, which depletes $\mathrm{Gr} 1^{+}$PMNs and monocytes in spleen and $\mathrm{PB}$, but not in BM (39). Depletion of the $\mathrm{Gr} 1^{+}$cells in the periphery resulted in a significant increase in $\mathrm{Gr} 1^{+} \mathrm{IMC}$ proliferation and subsequent accumulation of these cells in BM, and spleen later on. This mechanism could promote expansion of MDSCs. Our hypothesis was further supported by experiments demonstrating that Dr5 KO MDSCs had substantially better survival than WT MDSCs, which was associated with significantly decreased proliferation of MDSCs.

The data on poor MDSC survival were counterintuitive in light of our findings regarding MDSC accumulation and were different from previous reports showing decreased apoptosis in MDSCs $(10,12)$. The main difference of our study was that we traced the fate of MDSCs in vivo using BrdU labeling and adoptive transfer and assessed apoptosis of MDSCs without isolation of cells in spleen sections. Isolation of cells from spleens involving red cell lyses, use of magnetic beads, or sorting usually results in loss of some MDSCs. If these cells were in advanced stages of apoptosis, this would likely result in enrichment for cells with less apoptosis at the time of evaluation. In our study, when cells were cultured in vitro for as little as 3 hours, apoptosis in MDSCs became much more prominent than in cells with the same phenotype isolated from healthy mice. Hu and colleagues measured apoptosis of MDSCs after 24 hours culture and showed that it was slightly lower that in control cells (10). However, usually less than $20 \%$ of MDSCs survived 24 hours of culture, in contrast to $40 \%$ of
IMCs with the same phenotype from control mice. In this situation, evaluation of apoptosis in remaining cells likely would lead to skewed results. In their study, Sinha et al. did not address the spontaneous apoptosis or survival of MDSCs; they demonstrated that MDSCs are sensitive to FAS/FASL-induced apoptosis (12), which does not contradict our results.

We found that shorter survival of MDSCs was caused by caspase-8-dependent apoptosis via upregulation of DR5. Agonistic DR5 antibodies and recombinant TRAIL have been previously used to target tumor cells in several clinical trials with modest results $(9,16,17)$, partially attributed to the variability of TRAIL$\mathrm{R}$ expression in tumor cells. Myeloid cells, although expressing TRAIL, have low TRAIL-R expression and thus are resistant to killing by recombinant TRAIL (40). Our data indicated that targeting DR5 in mice did not affect PMNs and IMCs, but effectively eliminated MDSCs. This resulted in significant antitumor effects in immunogenic tumors and markedly promoted antitumor effects of immune therapy using CTLA4 blockade in weakly immunogeneic tumors. In previous studies, treatment of mice bearing DR5-expressing tumors with agonistic MD5-1 mAb resulted in antitumor effects $(41,42)$. These effects were associated with the generation of antitumor immune responses, but whether it was the result of a direct antitumor effect of the antibody or was mediated via other mechanisms remained unclear. Our data demonstrated that targeting DR5 improved antitumor responses in mice bearing TRAIL-insensitive tumors and that these responses were mediated by $\mathrm{CD}^{+} \mathrm{T}$ cells. Thus, it is likely that the elimination of MDSCs by anti-DR5 antibody treatment facilitated the expansion and function of antitumor $\mathrm{CD}^{+} \mathrm{T}$ cells.

In cancer patients, PMN-MDSCs also had lower survival than PMNs, and agonistic DR5 antibody as well as recombinant TRAIL killed these cells in vitro while not affecting PMNs. However, the mechanism of this effect was somewhat different. PMN-MDSCs had the same DR5 expression as did PMNs, but substantially lower DCR1 and DCR2 expression. The role of decoy receptors in TRAIL-mediated signaling is poorly understood. However, DCR2 may provide antiapoptotic signals neutralizing TRAIL-R signaling, whereas DCR1 inhibits TRAIL-induced apoptosis by competing for TRAIL binding $(43,44)$. This may explain why human PMN-MDSCs, in contrast to PMNs, were sensitive to TRAIL.

What could cause the changes in TRAIL-Rs in MDSCs? In recent years, it has become apparent that MDSC accumulation requires 2 sets of signals: one induces emergency myelopoiesis and affects myeloid cell differentiation, the other activates MDSCs. The first group of signals is mediated primarily by growth factors such as GM-CSF and G-CSF, and the second by proinflammatory cytokines (IL-1 $\beta$, IL-6, IFN- $\gamma$, and IL-4) $(1,6,7)$. Coincidently, NF- $\mathrm{BB}$ triggered by many proinflammatory factors regulates TRAIL-R expression (45). To our surprise, neither TCM nor a number of cytokines known to be present in the tumor milieu were able to upregulate DR5 in mouse IMCs to mimic the effect observed in mouse MDSCs.

Since the ER stress response was previously implicated in the regulation of TRAIL-Rs (46), we tested its possible role in DR5 expression in myeloid cells. The ER stress response pathway is a complex network responsible for maintaining ER homeostasis and is initiated by 3 protein sensors, PERK, IRE1 and ATF6, each of which activates a branch of the ER stress response (47). It has been implicated in cell differentiation, glucose metabolism, and TLR signaling (30). CHOP is usually considered the main media- 
tor of ER stress response-induced apoptosis (26) and is a known transcription factor able to directly regulate DR5 expression $(10,27)$. Our experiments demonstrated that treatment of mouse IMCs and PMNs with the ER stress inducers tunicamycin and thapsigargin resulted in upregulation of DR5, recapitulating the effects observed in MDSCs. Treatment of human PMNs with ER stress inducers did not affect DR5, but markedly downregulated DCR1 and DCR2, similar to the changes observed in MDSCs from cancer patients. These data are consistent with a previous report showing that thapsigargin could decrease TRAIL decoy receptor expression in giant cell tumor of bone (48). Human TCM, in contrast to mouse TES, induced the ER stress response in PMNs. Inhibition of ER stress by TUDCA abrogated TCMinducible downregulation of TRAIL decoy receptors on human PMNs. The mechanism by which ER stress can downregulate TRAIL decoy receptors remains to be elucidated. Our present data demonstrated that MDSCs in TB mice and cancer patients exhibit markedly higher levels of ER stress response than their tumor-free mouse and healthy donor counterparts. Several factors could induce ER stress in MDSCs. MDSCs are known to have high level of ROS, one of the major inducers of the ER stress response (49). Lipids represent another potent ER stress inducer (50), and lipid accumulation was shown to be associated with DCs (51) and other myeloid cells (data not shown). The tumor microenvironment may provide an additional source of ER stress inducers associated with low $\mathrm{pH}$, adenosine release, and hypoxia. Stronger changes in TRAIL-R expression observed in human and mouse MDSCs from tumor sites compared with MDSCs from PB and spleen may support this conclusion. Whether ER stress directly contributes to the suppressive activity of MDSCs remains to be elucidated.

In conclusion, our results suggest a direct role of the ER stress response in the fate of MDSCs in TB hosts and implicate TRAILRs in this process (Figure 9E). Changed expression of TRAIL-R patterns (agonist or decoy) can distinguish MDSCs from normal PMNs and monocytes. Targeting TRAIL-Rs can result in rapid and marked selective depletion of these cells in cancer patients and possibly in other pathologic conditions. This approach can be explored in efforts to enhance the antitumor effect of various immunotherapeutic agents.

\section{Methods}

Human samples. Samples were collected from patients at H. Lee Moffitt Cancer Center and Helen F. Graham Cancer Center. PB was collected from 11 subjects with previously untreated stage II-IV NSCLC at H. Lee Moffitt Cancer Center and 6 patients with NSCLC at Helen F. Graham Cancer Center. In addition, $\mathrm{PB}$ and tumor tissues were collected from 5 patients with stage III HNC at H. Lee Moffitt Cancer Center. PB from 8 healthy donors was used as control.

All cell samples were analyzed within 3 hours of collection. PMN-MDSCs were evaluated in mononuclear fraction of $\mathrm{PB}$ after ficoll density gradient. Neutrophils were evaluated from the cell fraction remaining after removal of mononuclear cells. Cells were resuspended in PBS and loaded on a step density gradient (Percoll 63\% on top of Percoll 72\%) to separate PMNs in a monolayer between the 2 Percoll phases.

Mice and tumor model. C57BL/6 and Balb/c female mice (6-8 weeks old) were obtained from NCI. Mice were kept in pathogen-free conditions. For experiments with DR5-deficient mice, lethally irradiated congenic CD45.1 ${ }^{+}$ mice (NCI) were reconstituted with BM cells from $\operatorname{Dr} 5 \mathrm{KO}$ mice (19). To establish tumors, $5 \times 10^{5}$ tumor cells were injected s.c. into C57BL/ 6 or
Balb/c mice. This number of cells established a s.c. tumor $1-1.5 \mathrm{~cm}$ in diameter within 2-3 weeks of injection. EL4 and EG7 thymomas, LLC lung carcinoma, CT26 colon carcinoma, and 4T1 mammary carcinoma were obtained from ATCC. Tumor cells were cultured in DMEM (BioSource International) supplemented with $10 \% \mathrm{FBS}, 5 \mathrm{mM}$ glutamine, and $1 \%$ antibiotics (Invitrogen). KPC mice were described previously (52).

Mice were treated with $200 \mu \mathrm{g}$ anti-CD8 mAb (clone 53-6.72; BioXCell), $100 \mu \mathrm{g}$ anti-CTLA4 mAb (clone 9D9; BioXCell), $250 \mu \mathrm{g}$ anti-Gr1 mAb (clone RB6-8C5; BioXCell), or $100 \mu \mathrm{g}$ anti-DR5 mAb (clone MD5-1).

Cell isolation and culture. Single-cell suspensions were prepared from spleens and BM, red blood cells were removed using ammonium chloride lysis buffer, and $\mathrm{Gr}^{+}$or $\mathrm{Ly}_{6} \mathrm{G}^{+}$cells were isolated subsequently using magnetic beads isolation using biotinylated Gr1 antibody (eBiosciences) or Ly6G antibody (Miltenyi). Cells were purified using streptavidin beads and MACS column according to the manufacturer's recommendation (Miltenyi). Human PMN-MDSCs were isolated on gradient (33), followed by labeling with $\mathrm{CD} 15-\mathrm{PE} \mathrm{mAb}$ (BD Biosciences), and then separated using anti-PE beads and MACS column (Miltenyi). Tissues were first digested with collagenase D (Sigma), and then red blood cells were lysed. Cells were then culture in RPMI (Biosource International) supplemented with $10 \%$ FBS, $5 \mathrm{mM}$ glutamine, $25 \mathrm{mM}$ HEPES, $50 \mu \mathrm{M} \beta$-mercaptoethanol, and $1 \%$ antibiotics (Invitrogen). In some experiments, recombinant GM-CSF was added to the culture media at a concentration of $10 \mathrm{ng} / \mathrm{ml}$ (Invitrogen). To assess TRAIL-mediated killing, mouse cells were cultured in plates pre-coated with Protein A (Pierce) followed by coating with $10 \mu \mathrm{g} / \mathrm{ml} \mathrm{MD5}-1 \mathrm{mAb}$. Human cells were cultured in the presence of either $200 \mathrm{ng} / \mathrm{ml}$ anti-human DR5 agonistic mAb (R\&D) or $100 \mathrm{ng} / \mathrm{ml}$ recombinant TRAIL (R\&D). To inhibit TRAIL signaling, mouse cells were cultured in the presence of recombinant murine OPG (R\&D) at different concentrations.

Flow cytometry. mAbs specific for the mouse cell surface markers CD11b, CD11c, Gr1, Ly6G, and Ly6C and for the human cell surface markers CD11b, CD14, CD15, and CD33 were purchased from BD Biosciences. DR5-specific mAb was purchased from eBiosciences; DCR1- and DCR2specific antibodies were purchased from R\&D Systems. Cleaved caspase-3 intracellular staining was performed using BD Cytofix/Cytoperm kit and staining with antibody specific for cleaved caspase-3 according to the manufacturer's recommendation (BD Biosciences). Annexin V staining was performed in annexin $\mathrm{V}$ buffer (BD Biosciences) according to the manufacturer's recommendations. BrdU labeling in vivo was performed by injection of BrdU i.p. 4 hours prior to cell harvest, and BrdU staining was performed using a BrdU Flow kit (BD Biosciences) according to the manufacturer's recommendations. Flow cytometry data were acquired using a BD LSR II flow cytometer and analyzed using FlowJo software (Tree Star).

Western blotting. Cells were lysed in RIPA buffer (Sigma-Aldrich) in the presence of protease inhibitor cocktail (Sigma-Aldrich). Whole-cell lysates were subjected to $10 \%-12 \%$ SDS-PAGE and transferred to PVDF membranes. Membranes were probed with appropriate primary antibodies overnight at $4^{\circ} \mathrm{C}$ : antibodies against BCL-2, BCL-XL, CHOP (human), cleaved caspase-3, cleaved caspase-8, and cleaved caspase-9 were from Cell Signaling Technology Inc.; antibodies against CHOP (mouse) and spliced XBP1 were from Santa Cruz Biotechnology Inc.; antibodies against DCR1 and DCR2 were from Abcam. Membranes were washed and incubated 2 hours at room temperature with secondary antibody conjugated with horseradish peroxidase (Santa Cruz Biotechnology Inc.). Results were visualized by chemiluminescence detection using a commercial kit (Pierce). To confirm equal loading, membranes were stripped and reprobed with antibody against $\beta$-actin (Santa Cruz Biotechnology Inc.) or GAPDH (Cell Signaling Technology Inc.).

Quantitative RT-PCR. RNA was extracted with total RNA extraction kit (Omega Bio-tek). cDNA was synthesized (cDNA reverse transcriptase kit; Applied Biosystems), and PCR was performed in duplicate for each 
sample. To detect expression of cytokines and $\beta$-actin, PCR was performed with $12.5 \mu \mathrm{l}$ SYBR Master Mixture (Applied Biosystems) and the following primers: Dr5, $5^{\prime}$-TGCGTTGCTGCTTGCTGTGCT- $3^{\prime}$ and 5'-GGTCCTCTTGATGGGCTCTCCTCC-3'; Dcr 1, 5'-GGTTCCTCTTGCTGCTGCTGCTGA-3' and 5'-TCCGGTCACTGGTGGCAGAACAG-3'; Dcr2, 5'-GGAAATGGTGGCCGACTGCTCAG-3' and 5'-GGTACATGGGCG-

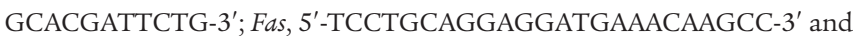
5'-GCTGGCAGCATTGATGGTGAGGT-3'; Tnfr1, 5'-GGCCACCTGGTCCGATCATCTTAC- $3^{\prime}$ and $5^{\prime}$-TGGATGTATCCCCATCAGCAGAGC-3'; Rank, 5'-ACTGAGGAGACCACCCAAGGAGGC-3' and 5'-ACCAGCACAACGGTCCCCTGAG-3'; Atf4, 5' -ATGGCCGGCTATGGATGAT-3' and 5'-CGAAGTCAAACTCTTTCAGATCCATT-3'; Bip, 5'-CATGGTTCTCACTAAAATGAAAGG-3' and 5'-GCTGGTACAGTAACAACT-3'; Chop, 5'-CTGCCTTTCACCTTGGAGAC-3' and $5^{\prime}$-CGTTTCCTGGGGATGAGATA-3'; Xbp 1, 5'-AAGAACACGCTTGGGAATGG-3' and 5'-ACTCCCCTTGGCCTCCAC-3'; Actb, 5'-ACCGCTCGTTGCCAATAGTGATGA-3' and 5'-TGAGAGGGAAATCGTGCGTGACAT-3'. Expressions of the different genes were normalized to $A c t b$. Relative expression was calculated using the $2^{-\Delta \Delta \mathrm{Ct}}$ method.

ChIP assay. ChIP was performed using the Acetyl-histone Immunoprecipitation Assay kit (Millipore) as previously described (13). The DNA was amplified by quantitative PCR using primers specific for the DR5 promoter sequence ( $5^{\prime}$-AGGTTAGTTCCGGTCCCTTC- 3 ' and 5'-CAACTGCAAATTCCACCACA-3').

Electron microscopy. More than $2 \times 10^{5}$ cells were fixed in $3 \%$ glutaraldehyde in $0.1 \mathrm{M}$ sodium phosphate buffer ( $\mathrm{pH} 7.4$ ) and embedded as a pellet in low gelling temperature agarose (Sigma-Aldrich). Cells were postfixed in $1 \%$ osmium tetroxide in $0.1 \%$ sodium phosphate buffer $(\mathrm{pH} 7.4)$ and stained in $2 \%$ uranyl acetate. The cells were washed with distilled water and dehydrated using an acetone gradient. They were gradually embedded in the araldite resin and cured for 18 hours at $65^{\circ} \mathrm{C}$. The ultrathin sections (100 nm thick) were collected on 200 mesh copper grids, stained with Reynold's lead citrate, and carbon coated. The grids of sections were viewed using a Jeol JEM-1200 EX electron microscope. At least 100 cells were counted per sample, cells were identified, and morphological differences were recorded. Cells were scored for the presence of dilated ER (indicative of ER stress), as follows: 1, cells containing dilated ER segments in up to one-third of the cytoplasm area; 2, cells containing dilated ER segments in over one-third and up to two-thirds of the cytoplasm area; 3 , cells containing dilated ER segments in over two-thirds of the cytoplasm area.

ELISPOT. The number of IFN- $\gamma$-producing T cells in response to cognate antigen was assessed in an ELISPOT assay (53). Each well contained $10^{5}$ cells. The number of spots was determined in triplicate using an automated ELISPOT counter (Cellular Technology).

Statistics. Unless otherwise indicated, data are presented as mean \pm SEM. Statistical analysis was performed using 2-tailed Student's $t$ test and GraphPad Prism 5 software (GraphPad Software Inc.). Tumor measurements were analyzed using 2-way ANOVA and 2-tailed $t$ test. A $P$ value less than 0.05 was considered significant.

Study approval. Human studies were approved by the Institutional Review Boards of the University of South Florida, the Christiana Care Health System at the Helen F. Graham Cancer Center, and The Wistar Institute. All patients signed approved consent forms. Animal studies were approved by The Wistar Institute and the University of South Florida Animal Care and Use Committees.

\section{Acknowledgments}

This work was supported in part by NIH grant CA084488 and developmental project from Moffitt lung SPORE grant P50 CA119997 to D.I. Gabrilovich. This work has been supported in part by the flow cytometry core facility at the H. Lee Moffitt Cancer Center and The Wistar Institute.

Received for publication October 31, 2013, and accepted in revised form March 20, 2014.

Address correspondence to: Dmitry I. Gabrilovich, The Wistar Institute, 3601 Spruce Street, Room 118, Philadelphia, Pennsylvania 19104, USA. Phone: 215.495.6955; Fax: 215.495.6948; E-mail: dgabrilovich@wistar.org.

Esteban Celis's present address is: Cancer Immunology, Inflammation and Tolerance Program, Georgia Regents University Cancer Center, Augusta, Georgia, USA.
1. Gabrilovich DI, Ostrand-Rosenberg S, Bronte $\mathrm{V}$. Coordinated regulation of myeloid cells by tumours. Nat Rev Immunol. 2012;12(4):253-268.

2. Youn JI, Nagaraj S, Collazo M, Gabrilovich DI. Subsets of myeloid-derived suppressor cells in tumorbearing mice. J Immunol. 2008;181(8):5791-5802.

3. Movahedi K, et al. Identification of discrete tumorinduced myeloid-derived suppressor cell subpopulations with distinct T-cell suppressive activity. Blood. 2008;111(8):4233-4244.

4. Youn JI, Collazo M, Shalova IN, Biswas SK, Gabrilovich DI. Characterization of the nature of granulocytic myeloid-derived suppressor cells in tumor-bearing mice. J Leukoc Biol. 2012;91(1):167-181.

5. Peranzoni E, et al. Myeloid-derived suppressor cell heterogeneity and subset definition. Curr Opin Immunol. 2010;22(2):238-244.

6. Condamine T, Gabrilovich DI. Molecular mechanisms regulating myeloid-derived suppressor cell differentiation and function. Trends Immunol. 2011; 32(1):19-25.

7. Sonda N, Chioda M, Zilio S, Simonato F, Bronte $\mathrm{V}$. Transcription factors in myeloid-derived suppressor cell generation. Curr Opin Immunol. 2011; 23(2):279-285.

8. Zhao X, et al. TNF signaling drives myeloid-derived suppressor cell accumulation. J Clin Invest. 2012; 122(11):4094-4104.

9. Roth F, De La Fuente AC, Vella JL, Zoso A, Inverardi
L, Serafini P. Aptamer-mediated blockade of IL4R $\alpha$ triggers apoptosis of MDSCs and limits tumor progression. Cancer Res. 2012;72(6):1373-1383.

10. Hu X, et al. Deregulation of apoptotic factors $\mathrm{Bcl}-\mathrm{xL}$ and Bax confers apoptotic resistance to myeloid-derived suppressor cells and contributes to their persistence in cancer. J Biol Chem. 2013; 288(26):19103-19115.

11. Chornoguz O, Grmai L, Sinha P, Artemenko KA, Zubarev RA, Ostrand-Rosenberg S. Proteomic pathway analysis reveals inflammation increases myeloid-derived suppressor cell resistance to apoptosis. Molecular \& cellular proteomics: MCP. 2011;10(3):M110 002980

12. Sinha P, Chornoguz O, Clements VK, Artemenko KA, Zubarev RA, Ostrand-Rosenberg S. Myeloidderived suppressor cells express the death receptor Fas and apoptose in response to T cell-expressed FasL. Blood. 2011;117(20):5381-5390.

13. Youn JI, et al. Epigenetic silencing of retinoblastoma gene regulates pathologic differentiation of myeloid cells in cancer. Nat Immunol. 2013;14(3):211-220.

14. Rudel T. Caspase inhibitors in prevention of apoptosis. Herz. 1999;24(3):236-241.

15. Danial NN, Korsmeyer SJ. Cell death: critical control points. Cell. 2004;116(2):205-219.

16. Johnstone RW, Frew AJ, Smyth MJ. The TRAIL apoptotic pathway in cancer onset, progression and therapy. Nature reviews. Cancer. 2008;8(10):782-798.
17. Abdulghani J, El-Deiry WS. TRAIL receptor signaling and therapeutics. Expert Opin Ther Targets. 2010; 14(10):1091-1108.

18. Emery JG, et al. Osteoprotegerin is a receptor for the cytotoxic ligand TRAIL. J Biol Chem. 1998; 273(23):14363-14367.

19. Finnberg N, et al. DR5 knockout mice are compromised in radiation-induced apoptosis. Mol Cell Biol. 2005;25(5):2000-2013.

20. Finnberg N, Klein-Szanto AJ, El-Deiry WS. TRAIL-R deficiency in mice promotes susceptibility to chronic inflammation and tumorigenesis. J Clin Invest. 2008;118(1):111-123.

21. Janssen EM, et al. CD4+ T-cell help controls CD8+ T-cell memory via TRAIL-mediated activationinduced cell death. Nature. 2005;434(7029):88-93.

22. Roberts AI, et al. The role of activation-induced cell death in the differentiation of T-helper-cell subsets. Immunol Res. 2003;28(3):285-293.

23. Pardoll DM. The blockade of immune checkpoints in cancer immunotherapy. Nat Rev Cancer. 2012; 12(4):252-264.

24. Cazanave SC, et al. Death receptor 5 signaling promotes hepatocyte lipoapoptosis. J Biol Chem. 2011;286(45):39336-39348.

25. Falschlehner C, Emmerich CH, Gerlach B, Walczak H. TRAIL signalling: decisions between life and death. Int J Biochem Cell Biol. 2007;39(7-8):1462-1475.

26. Tian X, Ye J, Alonso-Basanta M, Hahn SM, Koumenis 
C, Dorsey JF. Modulation of CCAAT/enhancer binding protein homologous protein (CHOP)-dependent DR5 expression by nelfinavir sensitizes glioblastoma multiforme cells to tumor necrosis factor-related apoptosis-inducing ligand (TRAIL). J Biol Chem. 2011;286(33):29408-29416.

27. Martin-Perez R, Niwa M, Lopez-Rivas A. ER stress sensitizes cells to TRAIL through down-regulation of FLIP and Mcl-1 and PERK-dependent up-regulation of TRAIL-R2. Apoptosis. 2012;17(4):349-363.

28. Tiwary R, Yu W, Li J, Park SK, Sanders BG, Kline $\mathrm{K}$. Role of endoplasmic reticulum stress in $\alpha$-TEA mediated TRAIL/DR5 death receptor dependent apoptosis. PLoS One. 2010;5(7):e11865.

29. Liu G, et al. Salermide up-regulates death receptor 5 expression through the ATF4-ATF3-CHOP axis and leads to apoptosis in human cancer cells. J Cell Mol Med. 2012;16(7):1618-1628.

30. Hetz C. The unfolded protein response: controlling cell fate decisions under ER stress and beyond. Nat Rev Mol Cell Biol. 2012;13(2):89-102.

31. Greten TF, Manns MP, Korangy F. Myeloid derived suppressor cells in human diseases. Int Immunopharmacol. 2011;11(7):802-807.

32. Montero AJ, Diaz-Montero CM, Kyriakopoulos CE, Bronte V, Mandruzzato S. Myeloid-derived suppressor cells in cancer patients: a clinical perspective. J Immunother. 2012;35(2):107-115.

33. Ko JS, et al. Sunitinib mediates reversal of myeloidderived suppressor cell accumulation in renal cell carcinoma patients. Clin Cancer Res. 2009; 15(6):2148-2157.

34. Ozcan U, et al. Chemical chaperones reduce ER stress and restore glucose homeostasis in a mouse model of type 2 diabetes. Science. 2006;313(5790):1137-1140.

35. Ilkovitch D, Lopez DM. The liver is a site for tumorinduced myeloid-derived suppressor cell accu- mulation and immunosuppression. Cancer Res. 2009;69(13):5514-5521.

36. Chen S, Akbar SM, Abe M, Hiasa Y, Onji M. Immunosuppressive functions of hepatic myeloid-derived suppressor cells of normal mice and in a murine model of chronic hepatitis B virus. Clin Exp Immunol. 2011;166(1):134-142.

37. Swirski FK, et al. Identification of splenic reservoir monocytes and their deployment to inflammatory sites. Science. 2009;325(5940):612-616.

38. Cortez-Retamozo V, et al. Origins of tumor-associated macrophages and neutrophils. Proc Natl Acad Sci US A. 2012;109(7):2491-2496.

39. Ribechini E, Leenen PJ, Lutz MB. Gr-1 antibody induces STAT signaling, macrophage marker expression and abrogation of myeloid-derived suppressor cell activity in BM cells. Eur J Immunol. 2009;39(12):3538-3551.

40. Walczak H, et al. Tumoricidal activity of tumor necrosis factor-related apoptosis-inducing ligand in vivo. Nature medicine. 1999;5(2):157-163.

41. Haynes NM, et al. CD11c dendritic cells and B cells contribute to the tumoricidal activity of anti-DR5 antibody therapy in established tumors. J Immunol. 2010;185(1):532-541.

42. Takeda K, et al. Induction of tumor-specific T cell immunity by anti-DR5 antibody therapy.J Exp Med. 2004;199(4):437-448.

43. Clancy L, et al. Preligand assembly domain-mediated ligand-independent association between TRAIL receptor 4 (TR4) and TR2 regulates TRAILinduced apoptosis. Proc Natl Acad Sci U S A. 2005; 102(50):18099-18104.

44. Merino D, Lalaoui N, Morizot A, Schneider P, Solary E, Micheau O. Differential inhibition of TRAILmediated DR5-DISC formation by decoy receptors 1 and 2. Mol Cell Biol. 2006;26(19):7046-7055.
45. Shetty S, et al. Transcription factor NF- $\mathrm{\kappa B}$ differentially regulates death receptor 5 expression involving histone deacetylase 1. Mol Cell Biol. 2005; 25(13):5404-5416.

46. Shiraishi T, et al. Tunicamycin enhances tumor necrosis factor-related apoptosis-inducing ligandinduced apoptosis in human prostate cancer cells. Cancer Res. 2005;65(14):6364-6370.

47. Walter P, Ron D. The unfolded protein response: from stress pathway to homeostatic regulation. Science. 2011;334(6059):1081-1086.

48. Huang L, Xu J, Li K, Zheng MH, Kumta SM. Thapsigargin potentiates TRAIL-induced apoptosis in giant cell tumor of bone. Bone. 2004; 34(6):971-981.

49. Santos CX, Tanaka LY, Wosniak J, Laurindo FR. Mechanisms and implications of reactive oxygen species generation during the unfolded protein response: roles of endoplasmic reticulum oxidoreductases, mitochondrial electron transport, and NADPH oxidase. Antioxid Redox Signal. 2009;11(10):2409-2427.

50. Fu S, Watkins SM, Hotamisligil GS. The role of endoplasmic reticulum in hepatic lipid homeostasis and stress signaling. Cell Metab. 2012;15(5):623-634.

51. Herber DL, et al. Lipid accumulation and dendritic cell dysfunction in cancer. Nat Med. 2010; 16(8):880-886.

52. Bayne LJ, et al. Tumor-derived granulocyte-macrophage colony-stimulating factor regulates myeloid inflammation and $\mathrm{T}$ cell immunity in pancreatic cancer. Cancer Cell. 2012;21(6):822-835.

53. Nagaraj S, Nelson A, Youn JI, Cheng P, Quiceno D, Gabrilovich DI. Antigen-specific CD4(+) T cells regulate function of myeloid-derived suppressor cells in cancer via retrograde MHC class II signaling. Cancer Res. 2012;72(4):928-938. 